This is the pre-copyedited version of "Energy-Minimizing, Symmetric Discretizations for Anisotropic Meshes and Energy Functional Extrapolation" first published in SIAM Journal on Scientific Computing 2021 43:4, A2448-A2473, published by the Society for Industrial and Applied Mathematics (SIAM). Unauthorized reproduction of this article is prohibited. Available online via https://epubs.siam.org/doi/abs/10.1137/21M1397520.

\title{
ENERGY-MINIMIZING, SYMMETRIC DISCRETIZATIONS FOR ANISOTROPIC MESHES AND ENERGY FUNCTIONAL EXTRAPOLATION
}

\author{
MARTIN JOACHIM KÜHN*† CAROLA KRUSE* ULRICH RÜDE*‡
}

\begin{abstract}
Self-adjoint differential operators often arise from variational calculus on energy functionals. In this case, a direct discretization of the energy functional induces a discretization of the differential operator. Following this approach, the discrete equations are naturally symmetric if the energy functional was self-adjoint, a property that may be lost when using standard difference formulas on nonuniform meshes or when the differential operator has varying coefficients. Low order finite difference or finite element systems can be derived by this approach in a systematic way and on logically structured meshes they become compact difference formulas. Extrapolation formulas used on the discrete energy can then lead to higher oder approximations of the differential operator. A rigorous analysis is presented for extrapolation used in combination with nonstandard integration rules for finite elements. Extrapolation can likewise be applied on matrix-free finite difference stencils. In our applications, both schemes show up to quartic order of convergence.
\end{abstract}

Key words. partial differential equation, energy functional, symmetry, anisotropy, extrapolation, finite differences, finite elements

AMS subject classifications. 65N06, 65N30, 65B99

1. Introduction. Self-adjoint differential operators are common in many applications. When discretizing such operators it is often essential to maintain the symmetry. This may have theoretical reasons when the eigenstructure of the matrix is of interest. Often there are also practical reasons, when solvers should be applied that rely on symmetry, see, e.g., [21]. In a finite element setting, symmetry is naturally preserved. However, for finite differences, a standard derivation of the discretization can lead to a nonsymmetric system matrix when the grid is irregular or when variable material parameters appear in the partial differential equation or the related energy functional $J(u)$.

For self-adjoint model problems, the construction of finite difference schemes that preserve symmetry is a classical topic; see, e.g., [25, p. $196 \mathrm{ff}$.$] or [6,1,24]$. In this paper, we present a natural approach to obtain symmetric finite difference stencils for anisotropic meshes by considering the energy functional corresponding to the partial differential equation similar as proposed in [25]. These stencils here lead to a novel approach to obtain a matrix-free implementation of the discretized operator. This is the key to reduce the memory footprint and in consequence it also helps to reduce the amount of data that must be transferred to the processing units for computing a matrix-vector multiplication. On many modern architectures, memory access bandwidth is limiting the performance of iterative solvers. With matrix-free methods the repeated loading of the system matrix of the discretized operator can be avoided.

Our approach is applicable to Cartesian meshes consisting of rectangular hexahedra or meshes with curvilinear tensor product structure. Such tensor product struc-

*Parallel Algorithms Team, CERFACS (Centre Européen de Recherche et de Formation Avancée en Calcul Scientifique), 42 Avenue Gaspard Coriolis, 31057 Toulouse Cedex 01, France. This project has received funding from the European Union's Horizon 2020 research and innovation programme under grant agreement No 824158 .

${ }^{\dagger}$ German Aerospace Center (DLR), Institute for Software Technology, Department for HighPerformance Computing, Cologne, Germany; Martin.Kuehn@DLR.de.

${ }^{\ddagger}$ Friedrich-Alexander Universität Erlangen-Nürnberg, Cauerstr. 11, 91058 Erlangen, Germany.

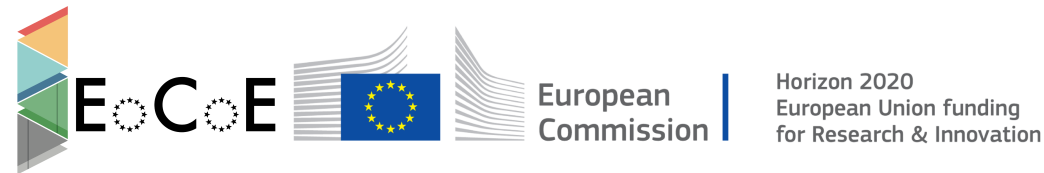


ture meshes appear in applications where a logically rectangular mesh is subjected to a curvilinear transformation. Typical examples are three-dimensional meshes in cylindrical coordinates or two-dimensional meshes in polar coordinates; cf. Figure 2.1. More general curvilinear coordinate systems are also possible, see, e.g., Figure 5.1.

On a family of successively finer meshes, the finite element or finite difference solution will converge to the solution of the differential equation. Under certain conditions, this limit process is governed by asymptotic error expansions, whose dominating terms can be eliminated by extrapolation $[4,16]$ to obtain results with higher order accuracy. The combination of extrapolation with multilevel and multigrid solvers seems in many ways natural and has thus recently seen renewed interest [17, 5, 23]. However, classical extrapolation techniques for boundary value problems depend on the regularity of the solution which limits their applicability. Therefore, so-called implicit variants of extrapolation have been developed $[20,19,10]$. These methods are related to the so-called $\tau$-extrapolation, a technique that has been proposed in the combination with multigrid solvers $[9,3]$. The theoretical justification of these techniques does not depend on the global regularity of the solution so that a wider range of applicability is possible.

On particular application of our here presented approach arises in the context of Tokamak fusion plasma modeled in Gyrokinetic codes such as Gysela [8]; see also our follow-up paper on the connection and application in detail [14].

In this paper, we will present an implicit extrapolation strategy that combines nodal finite element functions or finite difference discretizations for different meshing parameters with the goal to increase the resulting order of convergence on curvilinear and anisotropic meshes. These methods can then be naturally combined with fast multigrid solvers. For finite elements, we use a nonstandard integration rule from [15, 11] to show equality between an extrapolated stiffness matrix from linear nodal basis functions and the stiffness matrix from a quadratic nodal basis set. An extrapolated finite difference scheme will be derived from the underlying energy functional by extrapolating the discretized form of the functional. Here, the discretized functional leads to symmetric matrices that can be combined analogously as the stiffness matrices for finite elements in an attempt to eliminate the dominating error terms. Numerically, we will see that the stencils derived and extrapolated in this form also yield improved convergence rates when combined equivalently to their corresponding finite element counterpart.

2. Model problem and localized energy expressions. For a given domain $\Omega \in \mathbb{R}^{d}, d=2,3$, the well-known model problem of finding a solution of the partial differential equation

$$
-\nabla \cdot(A \nabla u)=f \quad \text { in } \Omega,
$$

can be traced back to the energy minimizing problem of

$$
\min _{u} J(u) ; \quad J(u):=\int_{\Omega} \frac{1}{2} \nabla u^{T} A \nabla u-f u \mathrm{~d} x,
$$

where $u$ comes from a suitable space and where certain boundary conditions such as $u=0$ on $\partial \Omega$ are imposed. Additionally, $f: \Omega \rightarrow \mathbb{R}$ and $A=\left(\begin{array}{cc}\alpha_{11} & \alpha_{12} \\ \alpha_{12} & \alpha_{22}\end{array}\right): \mathbb{R}^{2} \rightarrow \mathbb{R}^{2}$ is a symmetric, continuously bounded positive definite diffusion tensor with eigenvalues $\lambda_{1}, \lambda_{2}$ such that $0<\lambda_{L} \leq \lambda_{1}, \lambda_{2}<\lambda_{H}<\infty$. 


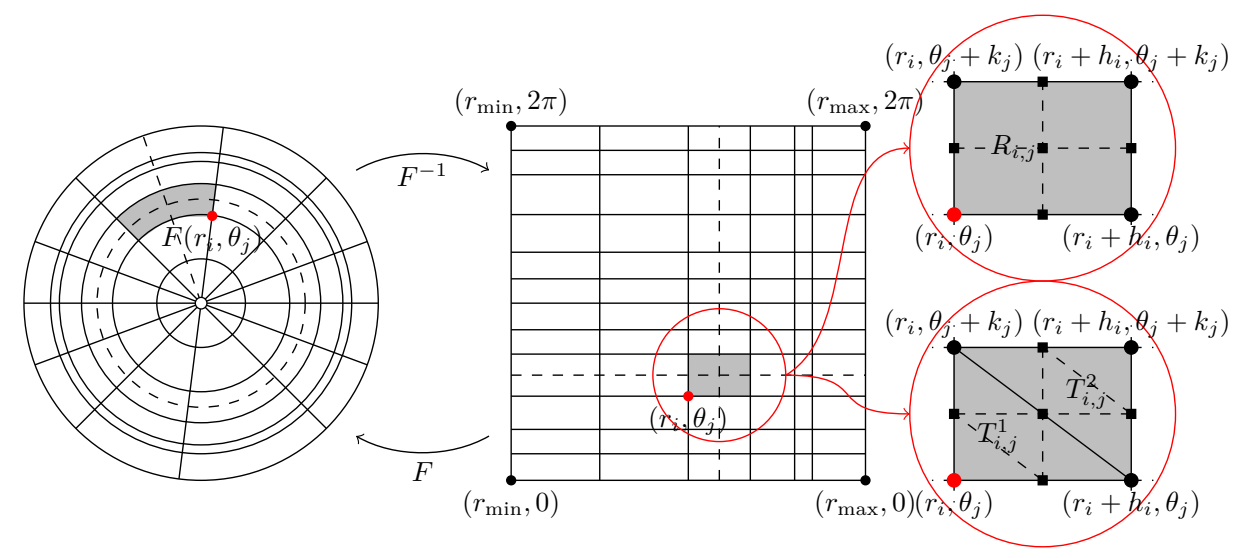

Figure 2.1. Physical (left) and logical (center) representation of an annulus, with an anisotropic, tensor product structured mesh, using polar coordinates. Mesh element $R_{i, j}=\left[r_{i}, \theta_{j}\right] \times\left[r_{i}+\right.$ $\left.h_{i}, \theta_{j}+k_{j}\right]$ colored in gray in both meshes; close-up view (right) with further subdivision into two triangles (bottom right). Additional regular subdivision of a mesh element, as used for our extrapolation method from section 4 , indicated by dashed lines (left to right) and quadratic markers for intermediate nodes (right).

In order to derive finite difference stencils that lead to symmetric linear systems, we consider the energy expression (2.2) element by element. Here, we assume a mesh of rectangular hexahedrals in three dimensions or rectangles in two dimensions which can be extended to a mesh of tensor product structure. Prominent examples of tensor product structured domains and meshes arise from problems posed in curvilinear coordinates such as polar coordinates. They are used to describe physical domains with curved coordinate lines by a logical domain of, often, Cartesian coordinates as illustrated in Figure 2.1 (left and center). 2D curvilinear coordinates can also be used to describe the cross sections of more complicated three-dimensional geometries. An example motivating this method in this article in particular, is the Tokamak geometry used in plasma fusion $[2,28]$.

We will restrict our study to arbitrary curvilinear transformations of an anisotropic Cartesian mesh in two dimensions, but the approach can be easily extended to the three-dimensional case. In the following, our presentation will consider the case of a (deformed) annulus described by the physical description $\Omega:=F\left(\Omega_{\ell}\right)$, given by an invertible function $F: \Omega_{L} \rightarrow \Omega$ such that $D F$ exists almost everywhere, $F(r, \theta)=(x, y)$, and a logical domain $\Omega_{\ell}=\left(r_{\min }, r_{\max }\right) \times[0,2 \pi)$, where $r_{\min }>0$. As the deformed annulus is just a pleasant representation of a tensor structured mesh, we exclude $r_{\min }=0$ here. Note that $\left(r_{\min }, r_{\max }\right) \times 0$ effectively represents interior points of the domain so that we enforce periodic boundary conditions thereon. The following study, however, remains also valid in case of Cartesian coordinates and/or Dirichlet or Neumann boundary conditions.

The resulting anisotropic mesh is given by the nodes $\left(r_{i}, \theta_{j}\right) \in \bar{\Omega}_{L}$ with

$$
\begin{aligned}
r_{1}:=r_{\min }, r_{n_{r}}:=r_{\max }, & r_{i+1}:=r_{i}+h_{i}, h_{i}>0,1 \leq i<n_{r} \\
\theta_{1}:=0, \theta_{n_{\theta}}:=2 \pi, & \theta_{j+1}:=\theta_{j}+k_{j}, k_{j}>0,1 \leq j<n_{\theta} .
\end{aligned}
$$


We denote $h:=\max _{i} h_{i}$ and $k:=\max _{j} k_{j}$ and introduce the notation

$$
v_{i+q_{1}, j+q_{2}}:=v\left(r_{i}+q_{1} h_{i}, \theta_{j}+q_{2} k_{j}\right)
$$

for any function $v$ depending on $(r, \theta)$ and $q_{1}, q_{2} \in[-1,1]$.

Remark 1. Due to our anisotropic meshes, we may have $h_{s} \neq h_{s-1}$ and $k_{t} \neq$ $k_{t-1}$. Our notation has to be understood as

$$
v_{(i-1)+q, j}=v\left(r_{i-1}+q h_{\mathbf{i}-\mathbf{1}}, \theta_{j}\right)
$$

and not as $v_{(i-1)+q, j}=v\left(r_{i-1}+q h_{i}, \theta_{j}\right)$. The subscript $(i-1)+q$ is thus understood as an index only, not as a computation. The same holds for the second variable.

Using the mapping $F$, each mesh element $R_{i, j}=\left[r_{i}, r_{i}+h_{i}\right] \times\left[\theta_{j}, \theta_{j}+k_{j}\right]$ of the logical domain $\bar{\Omega}_{L}$ has its curvilinear representation in $F\left(R_{i, j}\right) \subset \bar{\Omega}$; see Figure 2.1 for a simple example. After subdivision of $R_{i, j}$ into two triangles $T_{i, j}^{1}$ and $T_{i, j}^{2}$ (see Figure 2.1; bottom right), these triangles are naturally represented by $F\left(T_{i, j}^{m}\right), m=1,2$.

By transformation, we obtain

$$
\begin{aligned}
J_{R_{i, j}}(u): & =\int_{F\left(R_{i, j}\right)}\left(\frac{1}{2} \nabla u^{T} A \nabla u-f u\right) \mathrm{d}(x, y) \\
& =\int_{R_{i, j}}\left(\frac{1}{2}\left(A D F^{-T}\left(\begin{array}{c}
u_{r} \\
u_{\theta}
\end{array}\right), D F^{-T}\left(\begin{array}{l}
u_{r} \\
u_{\theta}
\end{array}\right)\right)-f u\right)|\operatorname{det} D F| \mathrm{d}(r, \theta)
\end{aligned}
$$

The functional arguments are $A=A(F(r, \theta)), D F^{-T}=D F^{-T}(F(r, \theta))$, and $\operatorname{det} D F=$ $\operatorname{det} D F(r, \theta)$. Note that in principle we have to distinguish $u(r, \theta)$ and $\widetilde{u}(x, y)$. We refrain from doing so to not overload the notation, the variables should become clear from the context.

In order to simplify the notation, we define

$$
\frac{1}{2} D F^{-1}(F(r, \theta)) A(F(r, \theta)) D F^{-T}(F(r, \theta))|\operatorname{det} D F(r, \theta)|=:\left(\begin{array}{cc}
a^{r r} & \frac{1}{2} a^{r \theta} \\
\frac{1}{2} a^{\theta r} & a^{\theta \theta}
\end{array}\right)
$$

and obtain the localized, reformulated energy expressions on $R_{i, j}$ and $T_{i, j}^{m}, m=1,2$, respectively, as

$$
\begin{aligned}
J_{R_{i, j}}(u) & =\int_{R_{i, j}}\left(a^{r r} u_{r}^{2}+a^{r \theta} u_{r} u_{\theta}+a^{\theta \theta} u_{\theta}^{2}-f u|\operatorname{det} D F|\right) \mathrm{d}(r, \theta) \\
& =\sum_{m=1}^{2} \int_{T_{i, j}^{m}}\left(a^{r r} u_{r}^{2}+a^{r \theta} u_{r} u_{\theta}+a^{\theta \theta} u_{\theta}^{2}-f u|\operatorname{det} D F|\right) \mathrm{d}(r, \theta) \\
& =: J_{T_{i, j}^{1}}(u)+J_{T_{i, j}^{2}}(u) .
\end{aligned}
$$

Note that (2.5) is symmetric and thus $a^{r \theta}=\frac{1}{2} a^{r \theta}+\frac{1}{2} a^{\theta r}$. Additionally, note that $a^{r \theta}=0$ if the diffusion tensor was diagonal and the physical domain could be described by, e.g., a simple polar coordinates transformation.

Obviously, the global energy can be written as

$$
J(u)=\sum_{i=1}^{n_{r}-1} \sum_{j=1}^{n_{\theta}-1} J_{R_{i, j}}(u)=\sum_{i=1}^{n_{r}-1} \sum_{j=1}^{n_{\theta}-1} \sum_{m=1}^{2} J_{T_{i, j}^{m}}(u) .
$$



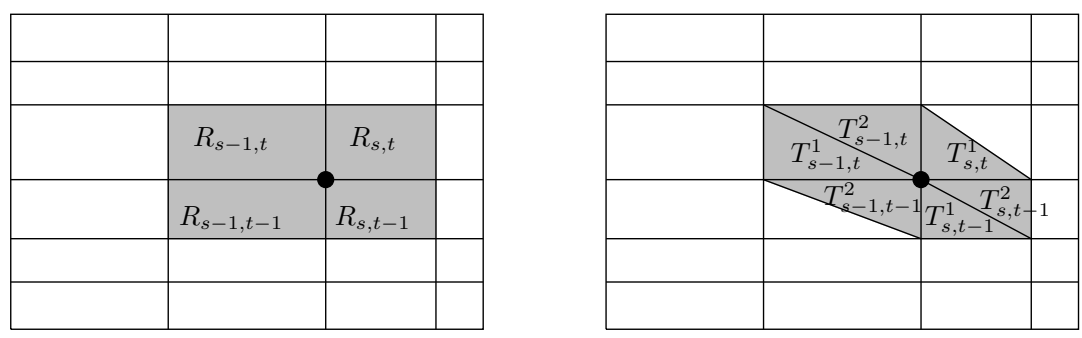

Figure 3.1. Mesh elements $R_{i, j}$ (left; five and nine point stencil) or $T_{i, j}^{1}$ and $T_{i, j}^{2}$ (right; seven point stencil), respectively, for which $u\left(r_{s}, \theta_{t}\right)$, for fixed but arbitrary $s$ and $t$, appears in the discretized, localized energy functional.

3. Discretization of local energy expressions with finite differences. Our approach to derive discretizations of the PDE is based on first discretizing the energy, i.e. the integral $J(u)$ represented by a sum of integrals over elements as in (2.7). Thus we now consider either the localized energy $J(u)$ on the mesh element $R_{i, j}$ or on $T_{i, j}^{1}$ and $T_{i, j}^{2}$. To obtain five-point or nine-point stencils, we consider rectangular elements. Triangles would have to be considered if seven-point stencils with support as illustrated in Figure 3.1 are derived. Note that for simple geometries and coefficients seven- or nine-point stencils may also degenerate to five-point stencils.

Numerical methods for integrals of the form (2.6) have been studied in [15] for the case of triangles and quadrilaterals. These methods are based on a combination of elementary finite difference formulas to approximate the derivatives and elementary numerical integration rules. Note that the discretization of these integrals is related to the process of computing the element stiffness matrices and assembling them to a global system. However, for deriving finite element discretizations, special shape functions are used. These shape functions are often polynomials so that they can be differentiated analytically, and the exact derivatives can be multiplied with the coefficients of the PDE. Numerical quadrature formulas are then applied in order to obtain the local element stiffness matrices.

The approach taken here differs from this standard finite element approach by using difference formulas to approximate the derivatives. At first sight replacing analytical derivatives by finite differences seems to introduce additional errors. As shown in [15], however, this does not necessarily lead to higher overall errors. Furthermore, as in [15], we will exploit that the combination of elementary difference and quadrature leads to approximations with asymptotic error expansions. This in turn invites the use of Richardson-type extrapolation. Note that this use of extrapolation differs from the classical one as e.g. analyzed in [16], where extrapolation is applied to numerical approximations of a PDE with different mesh sizes. In this case, extrapolation relies on the existence of expansion of the numerical PDE solution which in turn depends on the global regularity of the solution. In this article, we will use extrapolation implicitly, applied to the energy functional locally. Thus, extrapolation is used in this article to construct a higher order discretization by using higher order accuracy to compute the stiffness matrices. In practice, the improved discrete systems can be effectively constructed by combining low order difference stencils that originate from different mesh resolutions, similar as they are used in a multigrid algorithm. Different from conventional multigrid, we will use the hierarchy of different meshes here not only to accelerate the convergence of the linear solver, but additionally to raise the order of approximation. 
We proceed by introducing the difference operators

$$
\delta_{r, h} v(r, \theta):=\frac{v(r+h / 2, \theta)-v(r-h / 2, \theta)}{h}
$$

and

$$
\delta_{\theta, k} v(r, \theta):=\frac{v(r, \theta+k / 2)-v(r, \theta-k / 2)}{k},
$$

that are central $\mathcal{O}\left(h^{2}\right)$ and $\mathcal{O}\left(k^{2}\right)$ approximations for the derivatives $v_{r}$ and $v_{\theta}$, respectively, for all functions $v$ who satisfy the necessary smoothness assumptions. For constructing the necessary approximations, we additionally introduce the averaging operators

$$
\mu_{r, h} v(r, \theta):=\frac{v(r+h / 2, \theta)+v(r-h / 2, \theta)}{2}
$$

and

$$
\mu_{\theta, k} v(r, \theta):=\frac{v(r, \theta+k / 2)+v(r, \theta-k / 2)}{2}
$$

that constitute $\mathcal{O}\left(h^{2}\right)$ and $\mathcal{O}\left(k^{2}\right)$ accurate approximations to $v$ as before. Note that this is in analogy to definitions (3.6) and (3.5) of [15], respectively.

The application of a midpoint rule to integrate $a^{r r}(r, \theta) u_{r}^{2}(r, \theta)$ over $[r, r+h]$ (see (2.6)) formally requires a function evaluation at $r+h / 2$. Then, besides the straightforward

$$
a^{r r}\left(r+\frac{h}{2}, \theta\right) u_{r}^{2}\left(r+\frac{h}{2}, \theta\right)=a^{r r}\left(r+\frac{h}{2}, \theta\right)\left(\delta_{r, h} u\left(\left(r+\frac{h}{2}, \theta\right)\right)\right)^{2}+\mathcal{O}\left(h^{2}\right),
$$

the resulting approximation by quadrature can further be approximated by

$$
a^{r r}\left(r+\frac{h}{2}, \theta\right) u_{r}^{2}\left(r+\frac{h}{2}, \theta\right)=\mu_{r, h} a^{r r}\left(r+\frac{h}{2}, \theta\right)\left(\delta_{r, h} u\left(\left(r+\frac{h}{2}, \theta\right)\right)\right)^{2}+\mathcal{O}\left(h^{2}\right) .
$$

Further variants combining differencing using $\delta$ with averaging using $\mu$ are possible when the second variable $\theta$ is also considered .

Because of the differencing on $u$ and averaging applied to $a$, all the function evaluations are shifted to $r$ and $r+h$, similar as they would occur in a trapezoidal rule. Though at first sight the averaging on $a^{r r}$ seems to introduce additional errors, we use it here in a context that it has the effect of replacing a midpoint integration by a trapezoidal integration, both of which produce asymptotically the same error estimates. We also refer to [15] where it is shown that the averaging does not necessarily produce worse errors when developing cubature formulas for integrands containing derivatives. The averaging can also be seen as some linear interpolation.

Note that the roles of quadrature first and differencing/averaging second can also be inversed as long as the integrand is also defined accross the boundaries of the quadrilaterals.

Then, going one step further and using differencing and averaging in the $\theta$ direction, we can consider the integral over $R_{i, j}$. Evaluating the numerical approximation of the integral

$$
\int_{R_{i, j}} \mu_{\theta, h} \mu_{r, h} a^{r r}(r, \theta)\left(\mu_{r, \theta} \delta_{r, h} u(r, \theta)\right)^{2} \mathrm{~d}(r, \theta)
$$


by a tensor product midpoint rule (also called center rule in [15]) eventually only requires function values of $u$ and $a^{r r}$ at the corners of the rectangle. Note that this has been introduced as Discretization 2 already in [15].

Additionally, we can e.g. use [15, Theorem 3.5], showing that this approximation of the integral containing derivatives enjoys an even error expansion in powers of $h$, i.e. the odd error terms vanish. We also point to [15, Theorem 3.6], stating here that when $a^{r r}$ and $u_{r}$ are polynomials so that the original integrand $a^{r r} u_{r}^{2}$ is a polynomial of degree $p$, then the error expansion is finite with highest term of $\mathcal{O}\left(h^{p}\right)$ (in case of integrating over a rectangle).

Specifically, combining both theorems from [15], the approximation to the integral will be exact when $u$ and $a^{r r}$ are linear polynomials on $R_{i, j}$.

We next note that trivially the same techniques can be applied to integrands involving derivatives of $u$ in $\theta$ directions, while rather straightforward modifications are necessary for the mixed terms.

Function values of $u_{s, t}=u\left(r_{s}, \theta_{t}\right)$ are only needed from the adjacent mesh elements; see the indicated elements in Figure 3.1. For a more general discussion on the how to choose these values, we refer to the guidelines (G1-G3) discussed in [15]. With an appropriate choice of finite difference rule and the integration rule, all function values needed will lie in the support of the corresponding basis functions in the finite element counterpart. Eventually, defining $u=\left(u_{1,1}, u_{1,2}, \ldots, u_{2,1}, \ldots, u_{n_{r}, n_{\theta}-1}\right)$, we obtain a quadratic form

$$
\frac{1}{2} u^{T} E u-u^{T} f
$$

to represent the discretized energy. The numerical solution is characterized as minimizing this energy.

In order to minimize (3.5), we compute the zeros of the derivative with respect to $u$, leading to a system of linear equations. Note again that each $u_{s, t}$ only appears in few of the discretized summands of (2.7) so that the resulting system is sparse, see Figure 3.1 and subsection 3.1 for exemplary details.

3.1. A symmetric five point stencil. We expect the five point stencil to yield quadratic convergence only in the absence of mixed terms $u_{r} u_{\theta}$ in the energy functional. Therefore, we assume $a^{r \theta}=0$ in this section. This, e.g., holds when standard polar coordinates are used and when a diagonal diffusion tensor is considered. We now explicit one approach generically described in the previous section. We use the midpoint rule in $r$ for the first summand (with derivative in $r$ ) and the midpoint rule in $\vartheta$ for the second summand (with derivative in $\vartheta$ ). For the element $R_{i, j}=$ $\left[r_{i}, r_{i}+h_{i}\right] \times\left[\theta_{j}, \theta_{j}+k_{j}\right]$, we then obtain

$$
\begin{aligned}
J_{R_{i, j}}^{\mathrm{lhs}}(u):= & \int_{R_{i, j}}\left(a^{r r}(r, \theta) u_{r}^{2}(r, \theta)+a^{\theta \theta}(r, \theta) u_{\theta}^{2}(r, \theta)\right) \mathrm{d}(r, \theta) \\
= & h_{i} \int_{\theta_{j}}^{\theta_{j}+k_{j}} a^{r r}\left(r_{i}+\frac{h_{i}}{2}, \theta\right) u_{r}^{2}\left(r_{i}+\frac{h_{i}}{2}, \theta\right)+\mathcal{O}\left(h_{i}^{2}\right) \mathrm{d} \theta \\
& \quad+k_{j} \int_{r_{i}}^{r_{i}+h_{i}} a^{\theta \theta}\left(r, \theta_{j}+\frac{k_{j}}{2}\right) u_{\theta}^{2}\left(r, \theta_{j}+\frac{k_{j}}{2}\right)+\mathcal{O}\left(k_{j}^{2}\right) \mathrm{d} r .
\end{aligned}
$$

We then can replace the partial derivatives by using (3.1) and (3.2). If the coefficient $a^{r r}$ and $a^{\vartheta \vartheta}$ are known as continuous (and analytical) functions, one may evaluate the functions at center points. If we want to get rid of these intermediate 
evaluations, we use linear interpolation as given by the the averaging operators (3.3) and (3.4). Eventually, trapezoidal or midpoint rule is used to integrate the remaining integral terms. We then have

$$
\begin{aligned}
& J_{R_{i, j}}^{\mathrm{hhs}}(u)=h_{i} k_{j}[ \mu_{\theta, k}\left(a_{i+\frac{1}{2}, j+\frac{1}{2}}^{r r}\left(\delta_{r, h} u_{i+\frac{1}{2}, j+\frac{1}{2}}\right)^{2}\right) \\
&\left.+\mu_{r, h}\left(\mu_{\theta, k} a_{i+\frac{1}{2}, j+\frac{1}{2}}^{\theta \theta}\left(\delta_{\theta, k} u_{i+\frac{1}{2}, j+\frac{1}{2}}\right)^{2}\right)\right]+\mathcal{O}\left(h_{i}^{3} k_{j}+h_{i} k_{j}^{3}\right) \\
&=\frac{h_{i} k_{j}}{2}\left[a_{i+\frac{1}{2}, j+1}^{r r}\left(\frac{u_{i+1, j+1}-u_{i, j+1}}{h_{i}}\right)^{2}+a_{i+\frac{1}{2}, j}^{r r}\left(\frac{u_{i+1, j}-u_{i, j}}{h_{i}}\right)^{2}\right. \\
&+\frac{1}{2}\left(a_{i+1, j+1}^{\theta \theta}+a_{i+1, j}^{\theta \theta}\right)\left(\frac{u_{i+1, j+1}-u_{i+1, j}}{k_{j}}\right)^{2} \\
&\left.\quad+\frac{1}{2}\left(a_{i, j+1}^{\theta \theta}+a_{i, j}^{\theta \theta}\right)\left(\frac{u_{i, j+1}-u_{i, j}}{k_{j}}\right)^{2}\right]+\mathcal{O}\left(h_{i}^{3} k_{j}+h_{i} k_{j}^{3}\right) \\
&=: \widetilde{J}_{R_{i, j}}^{\mathrm{hs}}(u)+\mathcal{O}\left(h_{i}^{3} k_{j}+h_{i} k_{j}^{3}\right)
\end{aligned}
$$

Using the trapezoidal rule, we also obtain for $b=f u|\operatorname{det} D F|$

$$
\begin{aligned}
J_{R_{i, j}}^{\mathrm{rhs}}(u): & =\int_{r_{i}}^{r_{i}+h_{i}} \int_{\theta_{j}}^{\theta_{j}+k_{j}} b(r, \theta) \mathrm{d}(r, \theta) \\
& =h_{i} k_{j} \mu_{\theta, k} \mu_{r, h} b_{i+\frac{1}{2}, j+\frac{1}{2}}+\mathcal{O}\left(h_{i}^{3} k_{j}+h_{i} k_{j}^{3}\right) \\
& =\frac{h_{i} k_{j}}{4}\left(b_{i+1, j+1}+b_{i+1, j}+b_{i, j+1}+b_{i, j}\right)+\mathcal{O}\left(h_{i}^{3} k_{j}+h_{i} k_{j}^{3}\right) \\
& =\widetilde{J}_{R_{i, j}}^{\mathrm{rhs}}(u)+\mathcal{O}\left(h_{i}^{3} k_{j}+h_{i} k_{j}^{3}\right) .
\end{aligned}
$$

Using $h_{i} \leq h^{2}$ and $k_{j} \leq k^{2}, i, j \geq 1$, and the vector of function values $u=\left(u_{1,1}, u_{1,2}, \ldots, u_{2,1}, \ldots, u_{n_{r}, n_{\theta}-1}\right)$, we implicitly obtain the discrete energy operator $E$ such that

$$
\frac{1}{2} u^{T} E u-u^{T} f=\sum_{i=1}^{n_{r}-1} \sum_{j=1}^{n_{\theta}-1}\left(\widetilde{J}_{R_{i, j}}^{\mathrm{hss}}(u)+\widetilde{J}_{R_{i, j}}^{\mathrm{rhs}}(u)\right)
$$

has remainder term $\mathcal{O}\left(h^{2}+k^{2}\right)$.

REMARK 2. Although we allow for different discretizations in $r$ and $\theta$, we assume to have a $0<\tau<\infty$ such that $h=\tau k$.

In order to minimize (3.9), we have to compute the derivative of (3.9) with respect to $u$. However, we never explicitly compute $E$. Instead, we remark that for any interior point $\left(r_{s}, \theta_{t}\right)$ the derivative $\frac{\partial}{\partial u_{s, t}}$ only depends on four summands; cf Figure 3.1. Thus,

$$
\begin{aligned}
\frac{\partial}{\partial u_{s, t}}\left(\frac{1}{2} u^{T} E u-u^{T} f\right) & =\sum_{i=1}^{n_{r}-1} \sum_{j=1}^{n_{\theta}-1} \frac{\partial}{\partial u_{s, t}}\left(\widetilde{J}_{R_{i, j}}^{\mathrm{hs}}(u)+\widetilde{J}_{R_{i, j}}^{\mathrm{rhs}}(u)\right) \\
& =\sum_{(i, j) \in I_{s, t}} \frac{\partial}{\partial u_{s, t}}\left(\widetilde{J}_{R_{i, j}}^{\mathrm{hs}}(u)+\widetilde{J}_{R_{i, j}}^{\mathrm{rhs}}(u)\right),
\end{aligned}
$$


where $I_{s, t}:=\{(s, t),(s-1, t),(s, t-1),(s-1, t-1)\}$. For $(i, j)=(s, t)$, we have

$$
\begin{aligned}
& \frac{\partial}{\partial u_{s, t}}\left(\widetilde{J}_{R_{s, t}}^{\mathrm{hs}}(u)+\widetilde{J}_{R_{s, t}}^{\mathrm{hhs}}(u)\right)=\frac{h_{s} k_{t}}{2}\left[2 a_{s+\frac{1}{2}, t}^{r r}\left(\frac{u_{s+1, t}-u_{s, t}}{h_{s}}\right) \frac{-1}{h_{s}}\right. \\
& \left.\quad+\left(a_{s, t+1}^{\theta \theta}+a_{s, t}^{\theta \theta}\right)\left(\frac{u_{s, t+1}-u_{s, t}}{k_{t}}\right) \frac{-1}{k_{t}}\right]+\frac{h_{s} k_{t}}{4} f_{s, t}\left|\operatorname{det} D F_{s, t}\right| .
\end{aligned}
$$

Equivalently we obtain the derivatives for the expressions with $(i, j) \in I_{s, t} \backslash(s, t)$. The critical point condition

$$
\sum_{(i, j) \in I_{s, t}} \frac{\partial}{\partial u_{s, t}}\left(\widetilde{J}_{R_{i, j}}^{\mathrm{hs}}(u)+\widetilde{J}_{R_{i, j}}^{\mathrm{hs}}(u)\right)=0
$$

then yields, after reordering of the summands, a five point stencil, whose entries are

$$
\begin{aligned}
& \mathbf{u}_{\mathbf{s}+\mathbf{1}, \mathbf{t}}:\left(*_{5}\right)_{s+1, t}:=-\frac{k_{t}+k_{t-1}}{h_{s}} a^{r r}\left(r_{s}+\frac{h_{s}}{2}, \theta_{t}\right), \\
& \mathbf{u}_{\mathbf{s}-\mathbf{1 , t}}:\left(*_{5}\right)_{s-1, t}:=-\frac{k_{t}+k_{t-1}}{h_{s-1}} a^{r r}\left(r_{s-1}+\frac{h_{s-1}}{2}, \theta_{t}\right), \\
& \mathbf{u}_{\mathbf{s}, \mathbf{t}+\mathbf{1}}:\left(*_{5}\right)_{s, t+1}:=-\frac{h_{s}+h_{s-1}}{k_{t}} \frac{a^{\theta \theta}\left(r_{s}, \theta_{t}\right)+a^{\theta \theta}\left(r_{s}, \theta_{t}+k_{t}\right)}{2}, \\
& \mathbf{u}_{\mathbf{s}, \mathbf{t}-\mathbf{1}}:\left(*_{5}\right)_{s, t-1}:=-\frac{h_{s}+h_{s-1}}{k_{t-1}} \frac{a^{\theta \theta}\left(r_{s}, \theta_{t-1}\right)+a^{\theta \theta}\left(r_{s}, \theta_{t-1}+k_{t-1}\right)}{2}, \\
& \mathbf{u}_{\mathbf{s}, \mathbf{t}}:\left(*_{5}\right)_{s, t}:=-\left[\left(*_{5}\right)_{s+1, t}+\left(*_{5}\right)_{s-1, t}+\left(*_{5}\right)_{s, t+1}+\left(*_{5}\right)_{s, t-1}\right],
\end{aligned}
$$

with right hand side

$$
\frac{\left(h_{s}+h_{s-1}\right)\left(k_{t}+k_{t-1}\right)}{4} f_{s, t}\left|\operatorname{det} D F_{s, t}\right| .
$$

3.2. A symmetric nine point stencil. The nine point stencil is obtained similarly to the the five-point stencil in subsection 3.1, just by acknowledging $a^{r \theta} \neq 0$. We proceed by first applying the midpoint rule to evaluate the integrals, then we use (3.1)-(3.2) to approximate the derivatives and finally the averaging operators (3.3)(3.4) for approximating the remaining terms. We thus have function evaluations only at the corner points of the elements. We obtain

$$
\begin{aligned}
& J_{R_{i, j}}^{\mathrm{lhs}}(u):=\int_{R_{i, j}}\left(a^{r r} u_{r}^{2}+a^{r \theta} u_{r} u_{\theta}+a^{\theta \theta} u_{\theta}^{2}\right) \mathrm{d}(r, \theta) \\
& =h_{i} k_{j}\left[\mu_{\theta, k}\left(\mu_{r, h} a_{i+\frac{1}{2}, j+\frac{1}{2}}^{r r}\left(\delta_{r, h} u_{i+\frac{1}{2}, j+\frac{1}{2}}\right)^{2}\right)+\mu_{r, h}\left(\mu_{\theta, k} a_{i+\frac{1}{2}, j+\frac{1}{2}}^{\theta \theta}\left(\delta_{\theta, k} u_{i+\frac{1}{2}, j+\frac{1}{2}}\right)^{2}\right)\right] \\
& \quad+h_{i} k_{j} \mu_{\theta, k}\left(\mu_{r, h}\left(a_{i+\frac{1}{2}, j+\frac{1}{2}}^{r \theta} \delta_{\theta, k} u_{i+\frac{1}{2}, j+\frac{1}{2}}\right) \delta_{r, h} u_{i+\frac{1}{2}, j+\frac{1}{2}}\right)+\mathcal{O}\left(h_{i}^{3} k_{j}\right)+\mathcal{O}\left(h_{i} k_{j}^{3}\right) \\
& =\frac{h_{i} k_{j}}{4}\left[\left(a_{i+1, j}^{r r}+a_{i, j}^{r r}\right)\left(\frac{u_{i+1, j}-u_{i, j}}{h_{i}}\right)^{2}+\left(a_{i+1, j+1}^{r r}+a_{i, j+1}^{r r}\right)\left(\frac{u_{i+1, j+1}-u_{i, j+1}}{h_{i}}\right)^{2}\right] \\
& \quad+\frac{h_{i} k_{j}}{4}\left[\left(a_{i, j+1}^{\theta \theta}+a_{i, j}^{\theta \theta}\right)\left(\frac{u_{i, j+1}-u_{i, j}}{k_{j}}\right)^{2}+\left(a_{i+1, j+1}^{\theta \theta}+a_{i+1, j}^{\theta \theta}\right)\left(\frac{u_{i+1, j+1}-u_{i+1, j}}{k_{j}}\right)^{2}\right]
\end{aligned}
$$




$$
\begin{aligned}
& +\frac{h_{i} k_{j}}{4}\left[a_{i, j}^{r \theta}\left(\frac{u_{i, j+1}-u_{i, j}}{k_{j}}\right)+a_{i+1, j}^{r \theta}\left(\frac{u_{i+1, j+1}-u_{i+1, j}}{k_{j}}\right)\right]\left(\frac{u_{i+1, j}-u_{i, j}}{h_{i}}\right) \\
& +\frac{h_{i} k_{j}}{4}\left[a_{i, j+1}^{r \theta}\left(\frac{u_{i, j+1}-u_{i, j}}{k_{j}}\right)+a_{i+1, j+1}^{r \theta}\left(\frac{u_{i+1, j+1}-u_{i+1, j}}{k_{j}}\right)\right]\left(\frac{u_{i+1, j+1}-u_{i, j+1}}{h_{i}}\right) \\
& +\mathcal{O}\left(h_{i}^{3} k_{j}+h_{i} k_{j}^{3}\right) \\
= & : \tilde{J}_{R_{i, j}}^{\mathrm{hs}}(u)+\mathcal{O}\left(h_{i}^{3} k_{j}+h_{i} k_{j}^{3}\right)
\end{aligned}
$$

By following the same lines as in subsection 3.1 and equations (3.9)-(3.11) for our modified $\tilde{J}_{R_{i, j}}^{\text {lhs }}(u)$ and the same $\tilde{J}_{R_{i, j}}^{\text {rhs }}(u)$, we obtain a nine point stencil with the entries

$$
\begin{aligned}
& \mathbf{u}_{\mathbf{s}+\mathbf{1}, \mathbf{t}}:\left(*_{9}\right)_{s+1, t}:=-\frac{k_{t}+k_{t-1}}{h_{s}} \frac{a_{s, t}^{r r}+a_{s+1, t}^{r r}}{2} \\
& \mathbf{u}_{\mathbf{s}-\mathbf{1}, \mathbf{t}}:\left(*_{9}\right)_{s-1, t}:=-\frac{k_{t}+k_{t-1}}{h_{s-1}} \frac{a_{s-1, t}^{r r}+a_{s, t}^{r r}}{2} \\
& \mathbf{u}_{\mathbf{s}, \mathbf{t}+\mathbf{1}}:\left(*_{9}\right)_{s, t+1}:=-\frac{h_{s}+h_{s-1}}{k_{t}} \frac{a_{s, t}^{\theta \theta}+a_{s, t+1}^{\theta \theta}}{2} \\
& \mathbf{u}_{\mathbf{s}, \mathbf{t}-\mathbf{1}}:\left(*_{9}\right)_{s, t-1}:=-\frac{h_{s}+h_{s-1}}{k_{t-1}} \frac{a_{s, t-1}^{\theta \theta}+a_{s, t}^{\theta \theta}}{2} \\
& \mathbf{u}_{\mathbf{s + 1 , t + 1}}:\left(*_{9}\right)_{s+1, t+1}:=-\frac{a_{s+1, t}^{r \theta}+a_{s, t+1}^{r \theta}}{4} \\
& \mathbf{u}_{\mathbf{s}+\mathbf{1}, \mathbf{t}-\mathbf{1}}:\left(*_{9}\right)_{s+1, t-1}:=\frac{a_{s, t-1}^{r \theta}+a_{s+1, t}^{r \theta}}{4} \\
& \mathbf{u}_{\mathbf{s}-\mathbf{1}, \mathbf{t}+\mathbf{1}}:\left(*_{9}\right)_{s-1, t+1}:=\frac{a_{s-1, t}^{r \theta}+a_{s, t+1}^{r \theta}}{4} \\
& \mathbf{u}_{\mathbf{s}-\mathbf{1 , t}-\mathbf{1}}:\left(*_{9}\right)_{s-1, t-1}:=-\frac{a_{s-1, t}^{r \theta}+a_{s, t-1}^{r \theta}}{4} \\
& \mathbf{u}_{\mathbf{s}, \mathbf{t}}:\left(*_{9}\right)_{s, t}:=-\left[\left(*_{9}\right)_{s+1, t}+\left(*_{9}\right)_{s-1, t}+\left(*_{9}\right)_{s, t+1}+\left(*_{9}\right)_{s, t-1}\right]
\end{aligned}
$$

with right hand side as given in (3.14).

3.3. Symmetric seven point stencils. Symmetric seven point stencils with support as depicted in Figure 3.1 (right) are slightly more laborious to obtain. Instead of considering $R_{i, j}$, one considers $T_{i, j}^{1}$ and $T_{i, j}^{2}$ (see Figure 2.1 ; bottom right). We refer to [15, Sec. 4.2] for further details. If $A$ is symmetric positive definite, then the operator could also be rewritten to avoid the mixed terms as it was done for $[15$, Discretization 7].

4. Extrapolation methods based on a two-level hierarchical refinement. In the following we will present an extrapolation method based on [11] where intermediate nodes are introduced in the 2D grid. After some preliminary notation, we first provide the finite difference stencils and finite element discretizations used with extrapolation; see subsection 4.2, subsection 4.3, and subsection 4.4.

In subsection 4.4, we provide the concrete extrapolated stiffness matrices and right hand sides; in subsection 4.4.1, we give some additional theory, provided nonstandard integration rules are used with finite element discretizations.

Numerical results for finite difference stencils and finite elements with standard and nonstandard integration rules are given in section 5 . 

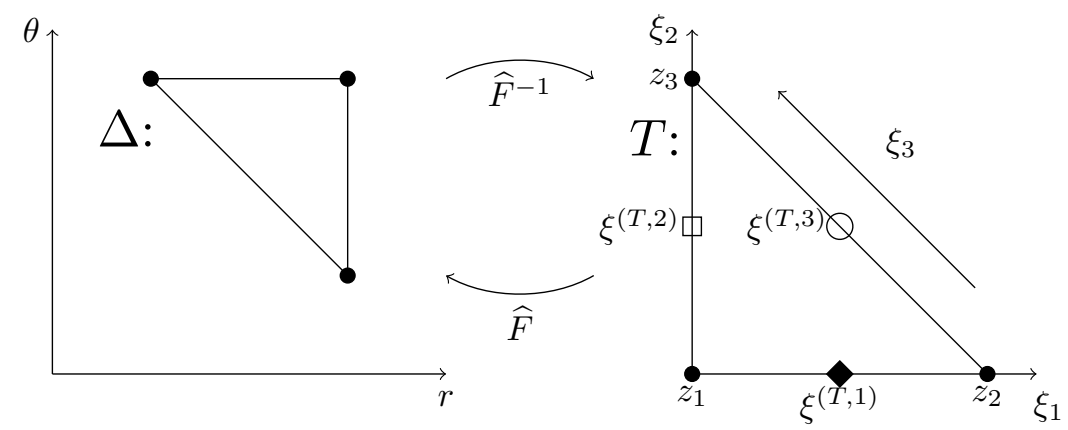

FIGURE 4.1. Mesh element $\Delta \in\left\{\mathcal{T}_{F}, \mathcal{T}_{C}\right\}$ (left) with transformation onto a reference triangle $T$ (right). Definition of the directions $\xi_{1}=e_{1}, \xi_{2}=e_{2}$, and $\xi_{3}=e_{2}-e_{1}$ as well as the definition of the evaluation nodes $\xi^{(T, 1)}, \xi^{(T, 2)}$, and $\xi^{(T, 3)}$ (right).

4.1. Two-level-hierarchical grids. Let us consider a two-level-hierarchical grid, i.e., for any anisotropic grid as given before, we incorporate all intermediate points $\left(r_{i+\frac{1}{2}}, \theta_{j}\right),\left(r_{i}, \theta_{j+\frac{1}{2}}\right)$, and $\left(r_{i+\frac{1}{2}}, \theta_{j+\frac{1}{2}}\right), 1 \leq i<n_{r}, 1 \leq j<n_{\theta}$, with

$$
r_{i+\frac{1}{2}}:=r_{i}+\frac{h_{i}}{2} \quad \text { and } \quad \theta_{j+\frac{1}{2}}:=\theta_{j}+\frac{k_{j}}{2}
$$

into the set of nodes on $\Omega_{\ell}$; see Figure 2.1. The coarse nodes are then given by $\left\{x_{1}, \ldots, x_{n_{c}}\right\}$. The new (logical) nodes are $\left\{x_{n_{c}+1}, \ldots, x_{n_{f}}\right\}$ and the refined mesh is given by $\left\{x_{1}, \ldots, x_{n_{f}}\right\}$. We denote the corresponding triangulations by $\mathcal{T}_{C}$ and $\mathcal{T}_{F}$.

4.2. Finite difference stencils. With the two-level-hierarchical grid given, we apply our compact five- or nine-point finite difference stencils from (3.13) or (3.16), respectively. We then obtain two stiffness matrices, generically denoted $K_{F}$ and $K_{C}$, on the fine triangulation $\mathcal{T}_{F}$ and on the coarse triangulation $\mathcal{T}_{C}$, respectively.

4.3. Finite elements with standard and nonstandard integration. Before introducing our choice of numerical integration, we formally define the nodal linear finite element spaces

$$
\begin{aligned}
V_{F}^{L}:=\left\{\varphi_{i}^{L} \in \mathcal{C}^{0}\left(\Omega_{\ell}\right): \varphi_{i}^{L}\left(x_{j}\right)=\delta_{i, j} ;\right. \\
\left.\varphi_{i}^{L} \in \mathcal{P}_{1}(\Delta) \forall \Delta \in \mathcal{T}_{F} ; 1 \leq i, j \leq n_{f}\right\} \\
\text { and } \quad V_{C}^{L_{C}}:=\left\{\varphi_{i}^{L_{C}} \in \mathcal{C}^{0}\left(\Omega_{\ell}\right): \varphi_{i}^{L_{C}}\left(x_{j}\right)=\delta_{i, j} ;\right. \\
\left.\varphi_{i}^{L_{C}} \in \mathcal{P}_{1}(\Delta) \forall \Delta \in \mathcal{T}_{C} ; 1 \leq i, j \leq n_{c}\right\}
\end{aligned}
$$

on $\mathcal{T}_{F}$ and $\mathcal{T}_{C}$, respectively.

Now, let $\Delta \in\left\{\mathcal{T}_{F}, \mathcal{T}_{C}\right\}$ and correspondingly $* \in\left\{L, L_{C}\right\}$. Consider two finite element basis functions $\varphi_{s}^{*}$ and $\varphi_{t}^{*}$ from the corresponding space defined in (4.1) 
which satisfy $\Delta \in \operatorname{supp}\left(\varphi_{s}^{*}\right) \cap \operatorname{supp}\left(\varphi_{t}^{*}\right)$. Then, we have

$$
\begin{aligned}
& \int_{\Delta}\left(\frac{\alpha}{2}\left(D F^{-T} \nabla_{r, \theta} \varphi_{s}^{*}, D F^{-T} \nabla_{r, \theta} \varphi_{t}^{*}\right)\right)|\operatorname{det} D F| \mathrm{d}(r, \theta) \\
= & \int_{T}\left(\frac{\alpha}{2}\left(D F^{-T} D \widehat{F}^{-T} \nabla_{\xi_{1}, \xi_{2}} \widehat{\varphi}_{\alpha}^{L_{C}}, D F^{-T} D \widehat{F}^{-T} \nabla_{\xi_{1}, \xi_{2}} \widehat{\varphi}_{\beta}^{L_{C}}\right)\right)|\operatorname{det} D F||\operatorname{det} D \widehat{F}| \mathrm{d}\left(\xi_{1}, \xi_{2}\right) \\
= & \int_{T}\left(\bar{b}^{\xi_{1} \xi_{1}} \frac{\partial \widehat{\varphi}_{\alpha}^{L_{C}}}{\partial \xi_{1}} \frac{\partial \widehat{\varphi}_{\beta}^{L_{C}}}{\partial \xi_{1}}+\bar{b}^{\xi_{1} \xi_{2}}\left(\frac{\partial \widehat{\varphi}_{\alpha}^{L_{C}}}{\partial \xi_{1}} \frac{\partial \widehat{\varphi}_{\beta}^{L_{C}}}{\partial \xi_{2}}+\frac{\partial \widehat{\varphi}_{\alpha}^{L_{C}}}{\partial \xi_{2}} \frac{\partial \widehat{\varphi}_{\beta}^{L_{C}}}{\partial \xi_{1}}\right)+\bar{b}^{\xi_{2} \xi_{2}} \frac{\partial \widehat{\varphi}_{\alpha}^{L_{C}}}{\partial \xi_{2}} \frac{\partial \widehat{\varphi}_{\beta}^{L_{C}}}{\partial \xi_{2}}\right) \mathrm{d}\left(\xi_{1}, \xi_{2}\right),
\end{aligned}
$$

where $\widehat{\varphi}_{\alpha}^{L_{C}}$ and $\widehat{\varphi}_{\beta}^{L_{C}}, \alpha, \beta \in\{1,2,3\}$, are the corresponding functions on the reference element $\widehat{F}^{-1}(\Delta)=T$; see Figure 4.1 and the appendix of our preprint [13]. As before, we have reduced the notation by defining

$$
\frac{\alpha}{2} D \widehat{F}^{-1} D F^{-1} D F^{-T} D \widehat{F}^{-T}|\operatorname{det} D F||\operatorname{det} D \widehat{F}|=:\left(\begin{array}{ll}
\bar{b}^{\xi_{1} \xi_{1}} & \bar{b}^{\xi_{1} \xi_{2}} \\
\bar{b}^{\xi_{1} \xi_{2}} & \bar{b}^{\xi_{2} \xi_{2}}
\end{array}\right) ;
$$

cf. (2.5). The functional arguments are $D F^{-T}=D F^{-T}\left(F\left(\widehat{F}\left(\xi_{1}, \xi_{2}\right)\right)\right)$, $\operatorname{det} D F=$ $\operatorname{det} D F\left(\widehat{F}\left(\xi_{1}, \xi_{2}\right)\right)$ as well as $D \widehat{F}^{-T}=D \widehat{F}^{-T}\left(\widehat{F}\left(\xi_{1}, \xi_{2}\right)\right)$, $\operatorname{det} D \widehat{F}=\operatorname{det} D \widehat{F}\left(\xi_{1}, \xi_{2}\right)$.

Standard numerical integration of the bilinear form. A standard rule of numerical integration, which is exact for polynomials $p \in \mathcal{P}_{2}$, is given by the Newton-Cotes formula

$$
\int_{T} g\left(\xi_{1}, \xi_{2}\right) \mathrm{d}\left(\xi_{1}, \xi_{2}\right)=\frac{|T|}{3} \sum_{i=1}^{3} g\left(\xi^{(T, i)}\right) ;
$$

see Figure 4.1 for a definition of $\xi^{(T, i)}, i=1,2,3$. In this paper, when refering to standard numerical integration, we always use (4.4) for (4.2).

Nonstandard numerical integration of the bilinear form [15, 11]. In order to introduce the nonstandard integration rules, we modify expression (4.2). As in [15, 11], we introduce the directional derivative

$$
\frac{\partial \varphi}{\partial \xi_{3}}=\frac{\partial \varphi}{\partial \xi_{2}}-\frac{\partial \varphi}{\partial \xi_{1}}
$$

and obtain after a short computation from the last line of (4.2), the expression

$$
\int_{T}\left(b^{\xi_{1} \xi_{1}} \frac{\partial \widehat{\varphi}_{\alpha}^{*}}{\partial \xi_{1}} \frac{\partial \widehat{\varphi}_{\beta}^{*}}{\partial \xi_{1}}+b^{\xi_{2} \xi_{2}} \frac{\partial \widehat{\varphi}_{\alpha}^{*}}{\partial \xi_{2}} \frac{\partial \widehat{\varphi}_{\beta}^{*}}{\partial \xi_{2}}+b^{\xi_{3} \xi_{3}} \frac{\partial \widehat{\varphi}_{\alpha}^{*}}{\partial \xi_{3}} \frac{\partial \widehat{\varphi}_{\beta}^{*}}{\partial \xi_{3}}\right) \mathrm{d}\left(\xi_{1}, \xi_{2}\right)
$$

by redefining

$$
b^{\xi_{1} \xi_{1}}:=\bar{b}^{\xi_{1} \xi_{1}}+\bar{b}^{\xi_{1} \xi_{2}}, b^{\xi_{2} \xi_{2}}:=\bar{b}^{\xi_{2} \xi_{2}}+\bar{b}^{\xi_{1} \xi_{2}} \text {, and } b^{\xi_{3} \xi_{3}}:=-\bar{b}^{\xi_{1} \xi_{2}} .
$$

Here, we have used a generic superindex $\cdot^{*}$ to no further define the basis functions $\widehat{\varphi}_{\alpha}^{*}$ and $\widehat{\varphi}_{\beta}^{*}$; which, in the sequel, can also be chosen from (4.11) instead of (4.1).

The nonstandard integration rule from $[15,11]$ then writes

$$
\begin{aligned}
& \int_{T}\left(b^{\xi_{1} \xi_{1}} \frac{\partial \widehat{\varphi}_{\alpha}^{*}}{\partial \xi_{1}} \frac{\partial \widehat{\varphi}_{\beta}^{*}}{\partial \xi_{1}}+b^{\xi_{2} \xi_{2}} \frac{\partial \widehat{\varphi}_{\alpha}^{*}}{\partial \xi_{2}} \frac{\partial \widehat{\varphi}_{\beta}^{*}}{\partial \xi_{2}}+b^{\xi_{3} \xi_{3}} \frac{\partial \widehat{\varphi}_{\alpha}^{*}}{\partial \xi_{3}} \frac{\partial \widehat{\varphi}_{\beta}^{*}}{\partial \xi_{3}}\right) \mathrm{d}\left(\xi_{1}, \xi_{2}\right) \\
& \approx \underbrace{|T|}_{=\frac{1}{2}} \sum_{n=1}^{3} b^{\xi_{n} \xi_{n}}\left(\xi^{(T, n)}\right) \frac{\partial \widehat{\varphi}_{\alpha}^{*}}{\partial \xi_{n}}\left(\xi^{(T, n)}\right) \frac{\partial \widehat{\varphi}_{\beta}^{*}}{\partial \xi_{n}}\left(\xi^{(T, n)}\right),
\end{aligned}
$$


where the evaluation points $\xi^{(T, n)}$ are defined in Figure 4.1.

REMARK 3. Geometrically and more generally spoken: we evaluate the partial derivative with respect to the direction $\xi_{n}$ and the coefficient $b^{\xi_{n} \xi_{n}}$ at the center node of the triangle edge which is parallel to the direction $\xi_{n}, n \in\{1,2,3\}$. This integration formula is only exact for constant summands in (4.8). Although $b_{n}^{\xi_{n} \xi_{n}}$ is not constant in our examples, we will see in section 5 that this formula yields cubic convergence as predicted when combined with extrapolation.

Numerical integration of the linear form. Independently of the integration of the bilinear form, the linear form will be approximated by the Newton-Cotes rule

$$
\int_{T} g\left(\xi_{1}, \xi_{2}\right) \mathrm{d}\left(\xi_{1}, \xi_{2}\right)=\frac{|T|}{3} \sum_{i=1}^{3} g\left(z_{i}\right)
$$

where $z_{i}, i \in\{1,2,3\}$, are the corner nodes as indicated in Figure 4.1. This numerical integration rule is exact for polynomials $p \in \mathcal{P}_{1}$.

4.4. Extrapolated matrices and vectors. In this section, we present our extrapolation strategy for finite differences and finite elements. We will focus on our compact finite difference stencils as summarized in subsection 4.2 as well as on nodal linear finite elements numerically integrated by one of the rules presented in subsection 4.3.

Given a discretization scheme on $\mathcal{T}_{F}$ as well as its corresponding scheme on $\mathcal{T}_{C}$, we assemble two stiffness matrices, generically denoted $K_{F}$ and $K_{C}$, on the fine and the coarse mesh, respectively. We introduce the extrapolated stiffness matrix and corresponding right hand side by

$$
K_{F}^{e x}:=\left(\begin{array}{cc}
\frac{4}{3} K_{F, c c}-\frac{1}{3} K_{C} & \frac{4}{3} K_{F, c f} \\
\frac{4}{3} K_{F, f c} & \frac{4}{3} K_{F, f f}
\end{array}\right) \quad \text { and } \quad f_{F}^{e x}:=\left(\begin{array}{c}
\frac{4}{3} f_{F, c}-\frac{1}{3} f_{C} \\
\frac{4}{3} f_{F, f}
\end{array}\right) .
$$

Here and in the following, the nodes, matrices, and vectors are ordered such that the coarse nodes, denoted by index ${ }_{c}$, come first and the intermediate fine nodes, denoted by index $\cdot_{f}$, come second.

In practice, we will see that this extrapolation step can increase the convergence order from two to three. From a theoretical point of view, we can prove that the sytem (4.10) is identical to one of quadratic basis functions if nonstandard numerical integration rules are used; see subsection 4.4.1.

REMARK 4. We remark that the use of quadratic basis functions versus linear ones will lead to better accuracy even if the solution exhibits regularity only locally. For example, in the case of a reentrant corner, the solution is typically smooth away from the singularity and the use of higher order elements there is well justified. Consequently, also the implicit extrapolation will be beneficial in this case, and this improvement of accuracy is achieved independently of the singularity is treated. Here, for example, methods as developed in [7, 18] could be used. We point out that this is in contrast to standard explicit Richardson-style extrapolation methods applied to PDE. Their justification relies on global asymptotic expansions. These expansions are affected by the so-called pollution effect caused by singular solutions at a reentrant corner that leads to a global error term of lower order. Standard Richardson extrapolation will only eliminate the higher order error terms, the global pollution error term will remain. Only if the global asymptotic error expansion exists and its order coefficients are known the explict extrapolation can also be modified such that it eliminates the pollution effect. This would have to be done for each reentrant corner singularity. 

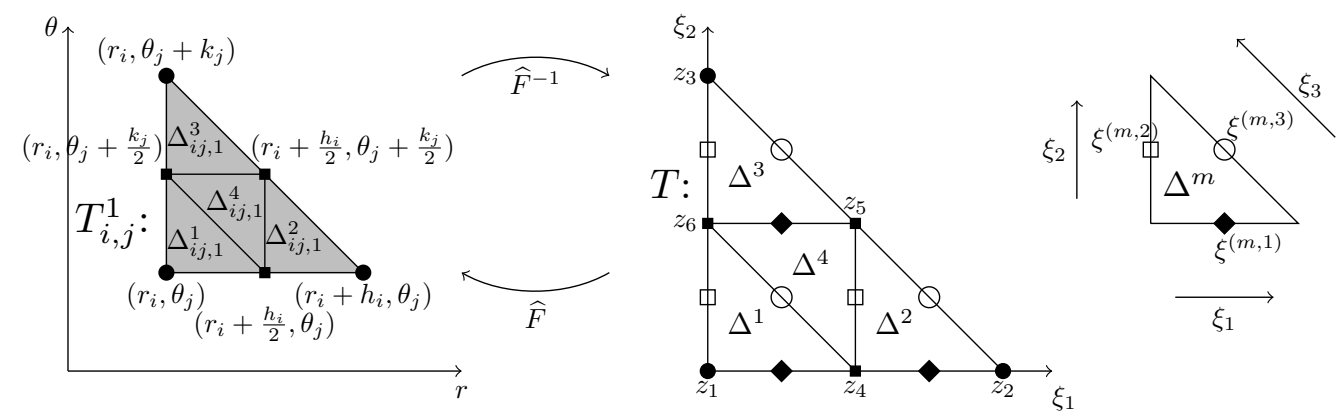

FIGURE 4.2. Coarse mesh element $T_{i, j}^{1}$ with subdivision into four subtriangles $\Delta_{i j, 1}^{m}, m=$ $1, \ldots, 4$, of equal size by taking intermediate points (left), transformation onto a reference triangle $T$ with four subtriangles $\Delta^{m}, m=1, \ldots, 4$ of size $\frac{1}{8}$ each (center). Close-up view of one subtriangle $\Delta^{m}$ of the reference element $T$ with definition of the directions $\xi_{1}=e_{1}, \xi_{2}=e_{2}$, and $\xi_{3}=e_{2}-e_{1}$ as well as the definition of the nodes $\xi^{(m, 1)}, \xi^{(m, 2)}$, and $\xi^{(m, 3)}, m=1, \ldots, 4$ (right).

4.4.1. Theoretical results for finite elements with nonstandard integration. In order to show some theoretical results, we have to introduce some additional finite element spaces. As our work is essentially based on [11], we analogously assume that our coefficients are smooth enough to justify higher order approximations at all. Additional spaces and related numerical integration rules. The nodal quadratic $\left(V_{F}^{Q}\right)$ as well as the two-level h- $\left(V_{F}^{H}\right)$ and p-hierarchical $\left(V_{F}^{P}\right)$ finite element spaces on the fine mesh are given by

$$
\begin{gathered}
V_{F}^{Q}:=\left\{\varphi_{i}^{Q} \in \mathcal{C}^{0}\left(\Omega_{\ell}\right): \varphi_{i}^{Q}\left(x_{j}\right)=\delta_{i, j} ;\right. \\
\left.\varphi_{i}^{Q} \in \mathcal{P}_{2}(\Delta) \forall \Delta \in \mathcal{T}_{F} ; 1 \leq i, j \leq n_{f}\right\}, \\
V_{F}^{H}:=V_{C}^{L_{C}} \cup\left\{\varphi_{i}^{H} \in \mathcal{C}^{0}\left(\Omega_{\ell}\right): \varphi_{i}^{H}\left(x_{j}\right)=\delta_{i, j} ;\right. \\
\left.\varphi_{i}^{H} \in \mathcal{P}_{1}(\Delta) \forall \Delta \in \mathcal{T}_{F} ; n_{c}+1 \leq i \leq n_{f} ; 1 \leq j \leq n_{f}\right\}, \\
V_{F}^{P}:=V_{C}^{L_{C}} \cup\left\{\varphi_{i}^{P} \in \mathcal{C}^{0}\left(\Omega_{\ell}\right): \varphi_{i}^{P}\left(x_{j}\right)=\delta_{i, j} ;\right. \\
\left.\varphi_{i}^{P} \in \mathcal{P}_{2}(\Delta) \forall \Delta \in \mathcal{T}_{F} ; n_{c}+1 \leq i \leq n_{f} ; 1 \leq j \leq n_{f}\right\},
\end{gathered}
$$

where $V_{C}^{L_{C}}$ was defined in (4.1).

For the spaces from (4.11), we use a different reference element representation. Instead of mapping each $\Delta \in \mathcal{T}_{F}$ onto the reference element, we map macro elements $\Delta \in \mathcal{T}_{C}$ such as $T_{i, j}^{n}=\bigcup_{m=1}^{4} \Delta_{i j, n}^{m}$ as defined in Figure 2.1, $n \in\{1,2\}$, onto a subdivided reference element $T=\bigcup_{m=1}^{4} \Delta^{m}$; see Figure 4.2.

For the generic macro element $T_{i, j}^{1}$, similar to (4.2), we consider

$$
\int_{T_{i, j}^{1}}\left(\frac{\alpha}{2}\left(D F^{-T} \nabla_{r, \theta} \varphi_{s}^{*}, D F^{-T} \nabla_{r, \theta} \varphi_{t}^{*}\right)\right)|\operatorname{det} D F| \mathrm{d}(r, \theta)
$$

where $\varphi_{s}^{*}$ and $\varphi_{t}^{*}$ are two finite element basis functions from a space defined in (4.11) with common support on $T_{i, j}^{1}$. In order to distinguish the different basis functions, we indicate $* \in\{Q, H, P\}$.

Nonstandard numerical integration of the bilinear form on $V_{F}^{H}$. For the linear $h$-hierarchical basis functions from $V_{F}^{H}$, the nonstandard integration of (4.12) 
writes

$$
\begin{aligned}
& \sum_{m=1}^{4} \int_{\Delta^{m}}\left(b^{\xi_{1} \xi_{1}} \frac{\partial \widehat{\varphi}_{\alpha}^{H}}{\partial \xi_{1}} \frac{\partial \widehat{\varphi}_{\beta}^{H}}{\partial \xi_{1}}+b^{\xi_{2} \xi_{2}} \frac{\partial \widehat{\varphi}_{\alpha}^{H}}{\partial \xi_{2}} \frac{\partial \widehat{\varphi}_{\beta}^{H}}{\partial \xi_{2}}+b^{\xi_{3} \xi_{3}} \frac{\partial \widehat{\varphi}_{\alpha}^{H}}{\partial \xi_{3}} \frac{\partial \widehat{\varphi}_{\beta}^{H}}{\partial \xi_{3}}\right) \mathrm{d}\left(\xi_{1}, \xi_{2}\right) \\
& \approx \sum_{m=1}^{4} \underbrace{\left|\Delta^{m}\right|}_{=\frac{1}{8}} \sum_{n=1}^{3} b^{\xi_{n} \xi_{n}}\left(\xi^{(m, n)}\right) \frac{\partial \widehat{\varphi}_{\alpha}^{H}}{\partial \xi_{n}}\left(\xi^{(m, n)}\right) \frac{\partial \widehat{\varphi}_{\beta}^{H}}{\partial \xi_{n}}\left(\xi^{(m, n)}\right),
\end{aligned}
$$

where $\widehat{\varphi}_{\alpha}^{H}$ and $\widehat{\varphi}_{\beta}^{H}, \alpha, \beta \in\{1, \ldots, 6\}$, are the corresponding basis functions on the subdivided reference element and where the evaluation points $\xi^{(m, n)}$ are defined in Figure 4.2. To obtain (4.13), we have again used (4.5), (4.6), and (4.7).

Numerical integration of the linear form on $V_{F}^{H}$. The right hand side expression will be approximated by applying the quadrature rule (4.9) on each micro element $\Delta^{m}, m \in\{1,2,3,4\}$.

Nonstandard numerical integration of the bilinear form on $V_{F}^{P}$ and $V_{F}^{Q}$. For the expressions related to $p$-hierarchical and quadratic basis functions, we use [11, eqs. (30)-(35)] to obtain the nonstandard integration rule of (4.12) as

$$
\begin{aligned}
\frac{1}{3} \underbrace{|T|}_{=\frac{1}{2}} \sum_{n=1}^{3}\left(\sum_{m=1}^{4} b^{\xi_{n} \xi_{n}}\left(\xi^{(m, n)}\right) \frac{\partial \widehat{\varphi}_{\alpha}^{*}}{\partial \xi_{n}}\left(\xi^{(m, n)}\right) \frac{\partial \widehat{\varphi}_{\beta}^{*}}{\partial \xi_{n}}\left(\xi^{(m, n)}\right)\right. \\
\left.-b^{\xi_{n} \xi_{n}}\left(\xi^{(T, n)}\right) \frac{\partial \widehat{\varphi}_{\alpha}^{*}}{\partial \xi_{n}}\left(\xi^{(T, n)}\right) \frac{\partial \widehat{\varphi}_{\beta}^{*}}{\partial \xi_{n}}\left(\xi^{(T, n)}\right)\right)
\end{aligned}
$$

where $\widehat{\varphi}_{\alpha}^{*}$ and $\widehat{\varphi}_{\beta}^{*}, \alpha, \beta \in\{1, \ldots, 6\}$, are the corresponding $p$-hierarchical $(*=P)$ or quadratic $(*=Q)$ basis functions on the subdivided reference element. Note that $z_{4}=\xi^{(T, 1)}, z_{5}=\xi^{(T, 2)}$, and $z_{6}=\xi^{(T, 3)}$; see Figure 4.2 and Figure 4.1. The quadrature formula (4.14) is exact for quadratic functions as already stated in [11].

Numerical integration of the linear form on $V_{F}^{P}$ and $V_{F}^{Q}$. The right hand side expression for $p$-hierarchical and quadratic basis functions will be obtained by the quadrature rule (4.4) on the entire reference element $T$ (i.e., without subdivision).

We denote the corresponding stiffness matrices on the spaces (4.1) and (4.11) and numerically approximated by either (4.8), (4.13), or (4.14) by $K_{F}^{L}, K_{C}^{L_{C}}, K_{F}^{H}, K_{F}^{P}$, $K_{F}^{Q}$ and proceed accordingly for the right hand sides and vectors on the corresponding spaces.

Theoretical results. First, we rephrase [11, Theorem 3.3] for the nodal bases from $V_{F}^{L}$ (and $V_{C}^{L_{C}}$ ) and $V_{F}^{Q}$. We will then show a relation between the nodal and the hierarchical approach as it was presented in [10] for the case of constant coefficients.

THEOREM 4.1. Let the stiffness matrices $K_{F}^{L}$ and $K_{F}^{L_{C}}$ be obtained by (4.8) and combined in $K_{F}^{L, e x}$ as given by (4.10). Furthermore, let the quadratic stiffness matrix $K_{F}^{Q}$ be obtained by (4.14). Let the right hand sides be accordingly obtained by (4.9) for the linear case and by (4.4) for the quadratic case; and $f_{F}^{L, e x}$ computed as in (4.10). Then, we have

$$
K_{F}^{L, e x}=K_{F}^{Q} \quad \text { and } \quad f_{F}^{L, e x}=f_{F}^{Q}
$$

and, trivially, both systems $K_{F}^{L, e x} u=f_{F}^{L, e x}$ and $K_{F}^{Q} u=f_{F}^{Q}$ yield the same solution. 
Proof. For the linear nodal basis, we map each triangle $\Delta=\Delta_{i, j}^{m} \in \mathcal{T}_{F}, m=$ $1, \ldots, 4$ onto the reference element $T$ without subdivsion. However, the nonstandard integration rules (4.8), (4.13), and (4.14) are consistent in the way that the directional derivatives are always evaluated on the triangle edge which is parallel to the direction; cf. also Remark 3. Except for the last term in (4.14), they are also always evaluated at the center node of the corresponding edge.

Since the element to element transformations are only affine transformations which keep parallel lines parallel, we can theoretically consider macro elements for the linear nodal basis functions. Instead of (4.8) for each $\Delta_{F} \subset \Delta_{C} \in \mathcal{T}_{C}$, we then consider (4.13) with basis functions $\widehat{\varphi}_{\alpha}^{L}$ and $\widehat{\varphi}_{\beta}^{L}, \alpha, \beta \in\{1, \ldots, 6\}$. For a compact sum presentation, we define $\hat{\varphi}_{\gamma}^{L_{C}}:=0$ for $\gamma>3$ although these functions do actually not exist; they are removed from the following formulas by the indicator function anyway.

In accordance to (4.10), we obtain the stiffness matrix $K_{F}^{L, e x}$ by

$$
\begin{aligned}
\sum_{n=1}^{3}\left(\frac{4}{3} * \frac{1}{8} \sum_{m=1}^{4} b^{\xi_{n} \xi_{n}}\left(\xi^{(m, n)}\right) \frac{\partial \widehat{\varphi}_{\alpha}^{L}}{\partial \xi_{n}}\left(\xi^{(m, n)}\right) \frac{\partial \widehat{\varphi}_{\beta}^{L}}{\partial \xi_{n}}\left(\xi^{(m, n)}\right)\right. \\
\left.-\frac{1}{3} I_{\leq 3}(\alpha, \beta) \frac{1}{2} b^{\xi_{n} \xi_{n}}\left(\xi^{(T, n)}\right) \frac{\partial \widehat{\varphi}_{\alpha}^{L_{C}}}{\partial \xi_{n}}\left(\xi^{(T, n)}\right) \frac{\partial \widehat{\varphi}_{\beta}^{L_{C}}}{\partial \xi_{n}}\left(\xi^{(T, n)}\right)\right)
\end{aligned}
$$

where $I_{\leq 3}(\alpha, \beta)$ is the indicator function

$$
I_{\leq 3}(\alpha, \beta)= \begin{cases}1, & \alpha, \beta \in\{1,2,3\} \\ 0, & \text { otherwise }\end{cases}
$$

Note that the second line in (4.16) accounts for the second summand in $K_{F}^{L, e x}$ which is only subtracted from the coarse block; cf. (4.10).

The proof of $K_{F}^{L, e x}=K_{F}^{Q}$ is only based on the comparison of functional values; cf. [11]. For the sake of convenience, we provide the values of the derivatives in the appendix of our preprint [13]. Considering (4.14) with $\widehat{\varphi}_{\alpha}^{Q}$ and $\widehat{\varphi}_{\beta}^{Q}, \alpha, \beta \in\{1, \ldots, 6\}$, and (4.16), we easily see from the appendix of our preprint [13] that the first parts are identical. The same applies to the expressions in the second lines since the directional derivatives of $\widehat{\varphi}_{\gamma}^{Q}, \gamma \in\{4,5,6\}$, are zero at $\xi^{(T, n)}, n \in\{1,2,3\}$.

The numerical integration rules (4.9) only uses node evaluations where the corresponding basis functions are either zero or one. We refrain from providing the values in an additional table. For the right hand side expression $f_{F}^{L, e x}$ and $\alpha \in\{1, \ldots, 6\}$, we consequently have

$$
\begin{aligned}
& \frac{4}{3} \sum_{m=1}^{4} \int_{\Delta^{m}} \bar{f} \varphi_{\alpha}^{L} \mathrm{~d}\left(\xi_{1}, \xi_{2}\right)-\frac{1}{3} I_{\leq 3}(\alpha, \alpha) \int_{T} \bar{f} \varphi_{\alpha}^{L_{C}} \mathrm{~d}\left(\xi_{1}, \xi_{2}\right) \\
& \approx\left\{\begin{array}{lll}
\frac{4}{3} * \frac{1}{24} \bar{f}\left(z_{\alpha}\right)-\frac{1}{3} * \frac{1}{6} \bar{f}\left(z_{\alpha}\right) & =0, & \alpha \in\{1,2,3\}, \\
\frac{4}{3} * \frac{3}{24} \bar{f}\left(z_{\alpha}\right) & =\frac{1}{6} \bar{f}\left(z_{\alpha}\right), & \alpha \in\{4,5,6\}
\end{array}\right.
\end{aligned}
$$

on the reference element. Here, $\bar{f}:=f|\operatorname{det} D F||\operatorname{det} D \widehat{F}|$ and $z_{\alpha}$ are defined as in Figure 4.2. Using (4.4) to obtain $f_{F}^{Q}$ yields the same values.

We now show a relation between the nodal approach using $K_{F}^{L, e x} u=f_{F}^{L, e x}$ and the hierarchical approach $K_{F}^{H, e x} u=f_{F}^{H, e x}$ using the nonstandard quadrature rules. Le us therefore introduce the transformation between the nodal and the hierarchical 
basis on the (logical) domain $\Omega_{\ell}$

$$
\begin{gathered}
T^{H}: V_{F}^{H} \rightarrow V_{F}^{L}, \quad T^{H}:=\left(\begin{array}{cc}
I_{c} & 0 \\
T_{f c}^{H} & I_{f}
\end{array}\right), \\
\left(T_{f c}^{H}\right)_{s, t}:= \begin{cases}\frac{1}{2}, & \text { if there exists an edge } e \text { in } \mathcal{T}_{C} \text { s.t. } x_{s} \in e \text { and } x_{t} \in \partial e, \\
0, & \text { otherwise. }\end{cases}
\end{gathered}
$$

Note that edges are open sets, i.e., $\stackrel{\circ}{e}=e$. The inverse transformation from nodal to hierarchical basis is

$$
T^{L}: V_{F}^{L} \rightarrow V_{F}^{H}, \quad T^{L}:=\left(\begin{array}{cc}
I_{c} & 0 \\
-T_{f c}^{H} & I_{f}
\end{array}\right) .
$$

Lemma 4.2. For the stiffness matrices $K_{F}^{L}$ and $K_{F}^{H}$ built from (4.8) and (4.13), respectively, and $\widetilde{K}_{F}^{L}=T^{L^{T}} K_{F}^{H} T^{L}$, we have

$$
K_{F}^{L}=\widetilde{K}_{F}^{L} .
$$

For the right hand sides $f_{F}^{L}$ and $f_{F}^{H}$ built from (4.9) and (4.4), respectively, and $\widetilde{f}_{F}^{L}=T^{L^{T}} f_{F}^{H}$, we have

$$
f_{F}^{L}=\widetilde{f}_{F}^{L} .
$$

REMARK 5. Lemma 4.2 seems obvious. However, for our nonstandard quadrature rules, it may not be clear that the coefficients $b^{\left(\xi_{i} \xi_{i}\right)}$ are evaluated implicitly at exactly the same nodes when $\widetilde{K}_{F}^{L}=T^{L^{T}} K_{F}^{H} T^{L}$ is computed and that the error in $K_{F}^{L}=\widetilde{K}_{F}^{L}$ is identical.

Proof. As in the proof of Theorem 4.1, we use the macro element for the theoretical assembly of $\widetilde{K}_{F}^{L}$. We have

$$
\widetilde{K}_{F}^{L}=\left(\begin{array}{cc}
K_{F, c c}^{H}-K_{F, c f}^{H} T_{f c}^{H}-T_{f c}^{H^{T}} K_{F, f c}^{H}+T_{f c}^{H^{T}} K_{F, f f}^{H} T_{f c}^{H} & K_{F, c f}^{H}-T_{f c}^{H^{T}} K_{F, f f}^{H} \\
K_{F, f c}^{H}-K_{F, f f}^{H} T_{f c}^{H} & K_{F, f f}^{H}
\end{array}\right) .
$$

In order to show the equality of the two matrix expressions, we always consider the corresponding computations on the reference element. On the reference element, the lower diagonal block of the transformation $\widehat{T}^{H}$ from the hierarchical to the nodal basis is

$$
\widehat{T}_{f c}^{H}=\left(\begin{array}{ccc}
\frac{1}{2} & \frac{1}{2} & 0 \\
0 & \frac{1}{2} & \frac{1}{2} \\
\frac{1}{2} & 0 & \frac{1}{2}
\end{array}\right)
$$

Note that the functions $\widehat{\varphi}_{\alpha}^{*}$ are associated with the nodes $z_{\alpha}, \alpha=1, \ldots, 6$; cf. Figure 4.2. For the nodal space, we have the basis functions $\left\{\widehat{\varphi}_{\alpha}^{L}\right\}_{\alpha=1, \ldots, 6}$, for the hierarchical space, we have the basis functions $\left\{\widehat{\varphi}_{\alpha}^{L_{C}}\right\}_{\alpha=1, \ldots, 3} \cup\left\{\widehat{\varphi}_{\alpha}^{L}\right\}_{\alpha=4, \ldots, 6}$.

Since the basis functions on $z_{4}, z_{5}$, and $z_{6}$ and the integration rules are identic on the macro element (cf. (4.8) and (4.13)), we trivially have

$$
K_{F, f f}^{L}=K_{F, f f}^{H} .
$$

The remaining part of the proof only relies on the values of the directional derivatives at the evaluation points given in Figure 4.2. 
We now consider the lower diagonal block to show $K_{F, f c}^{L}=K_{F, f c}^{H}-K_{F, f f}^{H} T_{f c}^{H}$. For symmetry reasons, this will also cover the upper diagonal block. A generic triangle share of $K_{F, f c}^{H}-K_{F, f f}^{H} T_{f c}^{H}$ computed on the reference triangle is given with $\alpha \in\{4,5,6\}$ and $\beta \in\{1,2,3\}$ by

$$
\frac{1}{8} \sum_{m=1}^{4} \sum_{n=1}^{3} b^{\xi_{n} \xi_{n}}\left(\xi^{(m, n)}\right) \frac{\partial \widehat{\varphi}_{\alpha}^{L}}{\partial \xi_{n}}\left(\xi^{(m, n)}\right)\left(\frac{\partial \widehat{\varphi}_{\beta}^{L_{C}}}{\partial \xi_{n}}\left(\xi^{(m, n)}\right)-\frac{1}{2} \sum_{\substack{\gamma \in\{4,5,6\} \\[\gamma, \beta] \subset e \tau_{C}}} \frac{\partial \widehat{\varphi}_{\gamma}^{L}}{\partial \xi_{n}}\left(\xi^{(m, n)}\right)\right) .
$$

For the additional condition on the sum, we used the shorthand notation

$$
\begin{aligned}
{[\gamma, \beta] \subset e_{\mathcal{T}_{C}}: \Leftrightarrow } & \text { The straigt line }\left[z_{\gamma}, z_{\beta}\right] \text { represents one half } \\
& \text { of the closure of an edge } e \text { in } \mathcal{T}_{C} ;
\end{aligned}
$$

cf. (4.17). For $\beta=1$, we exemplarily have $\gamma \in\{4,6\}$ and

$$
\frac{1}{8}\left(-2 b^{\xi_{1} \xi_{1}}\left(\xi^{(1,1)}\right) \frac{\partial \widehat{\varphi}_{\alpha}^{L}}{\partial \xi_{1}}\left(\xi^{(1,1)}\right)-2 b^{\xi_{2} \xi_{2}}\left(\xi^{(1,2)}\right) \frac{\partial \widehat{\varphi}_{\alpha}^{L}}{\partial \xi_{2}}\left(\xi^{(1,2)}\right)\right)=:(*)_{1, \alpha} ;
$$

see the third row and the rows -6 to -4 in the appendix of our preprint [13] for the evaluation of the parenthesis' expression. Many terms cancel each other out. For $\beta \in\{2,3\}$, we have

$$
\begin{array}{ll}
\beta=2: & \frac{1}{8}\left(2 b^{\xi_{1} \xi_{1}}\left(\xi^{(2,1)}\right) \frac{\partial \widehat{\varphi}_{\alpha}^{L}}{\partial \xi_{1}}\left(\xi^{(2,1)}\right)-2 b^{\xi_{3} \xi_{3}}\left(\xi^{(2,3)}\right) \frac{\partial \widehat{\varphi}_{\alpha}^{L}}{\partial \xi_{3}}\left(\xi^{(2,3)}\right)\right)=:(*)_{2, \alpha} \\
\beta=3: & \frac{1}{8}\left(2 b^{\xi_{2} \xi_{2}}\left(\xi^{(3,2)}\right) \frac{\partial \widehat{\varphi}_{\alpha}^{L}}{\partial \xi_{2}}\left(\xi^{(3,2)}\right)+2 b^{\xi_{3} \xi_{3}}\left(\xi^{(3,3)}\right) \frac{\partial \widehat{\varphi}_{\alpha}^{L}}{\partial \xi_{3}}\left(\xi^{(3,3)}\right)\right)=:(*)_{3, \alpha} .
\end{array}
$$

For $\alpha \in\{1, \ldots, 6\}$ and the product with the nodal basis functions on the fine grid $\widehat{\varphi}_{\beta}^{L}, \beta \in\{1,2,3\}$, we have

$$
\int_{T} \frac{1}{8} \sum_{n=1}^{3} b^{\xi_{n} \xi_{n}} \frac{\partial \widehat{\varphi}_{\alpha}^{L}}{\partial \xi_{n}} \frac{\partial \widehat{\varphi}_{\beta}^{L}}{\partial \xi_{n}} \mathrm{~d}\left(\xi_{1}, \xi_{2}\right)=(*)_{\beta, \alpha}
$$

For $\alpha \in\{4,5,6\}$, this yields the equality of the offdiagonal blocks in $K_{F}^{L}$ and $\widetilde{K}_{F}^{L}$.

We use the results of the previous step and write the upper left block as

$$
K_{F, c c}^{H}-K_{F, c f}^{H} T_{f c}^{H}-T_{f c}^{H^{T}}\left(K_{F, f c}^{H}-K_{F, f f}^{H} T_{f c}^{H}\right)=K_{F, c c}^{H}-K_{F, c f}^{H} T_{f c}^{H}-T_{f c}^{H^{T}} K_{F, f c}^{L} .
$$

First, we consider $T_{f c}^{H^{T}} K_{F, f c}^{L}$. Note that the preceeding expressions $(*)_{\beta, \alpha}$ are also valid for $\alpha \in\{1,2,3\}$ and correspond to the columns of $K_{F, f c}^{L}$ and the coarse nodes $z_{1}, z_{2}$, and $z_{3}$, respectively. We have

$$
\begin{aligned}
& T_{f c}^{H^{T}} K_{F, f c}^{L}=\frac{1}{2}\left(\begin{array}{ccc}
(*)_{1,4}+(*)_{1,6} & (*)_{2,4}+(*)_{2,6} & (*)_{3,4}+(*)_{3,6} \\
(*)_{1,4}+(*)_{1,5} & (*)_{2,4}+(*)_{2,5} & (*)_{3,4}+(*)_{3,5} \\
(*)_{1,5}+(*)_{1,6} & (*)_{2,5}+(*)_{2,6} & (*)_{3,5}+(*)_{3,6}
\end{array}\right) \\
& =-\frac{1}{4}\left(\begin{array}{ccc}
b^{\xi_{1} \xi_{1}}\left(\xi^{(1,1)}\right)+b^{\xi_{2} \xi_{2}}\left(\xi^{(1,2)}\right) & b^{\xi_{1} \xi_{1}}\left(\xi^{(2,1)}\right) & b^{\xi_{2} \xi_{2}}\left(\xi^{(3,2)}\right) \\
b^{\xi_{1} \xi_{1}}\left(\xi^{(1,1)}\right) & b^{\xi_{1} \xi_{1}}\left(\xi^{(2,1)}\right)+b^{\xi_{3} \xi_{3}}\left(\xi^{(2,3)}\right) & b^{\xi_{3} \xi_{3}}\left(\xi^{(3,3)}\right) \\
b^{\xi_{2} \xi_{2}}\left(\xi^{(1,2)}\right) & b^{\xi_{3} \xi_{3}}\left(\xi^{(2,3)}\right) & b^{\xi_{2} \xi_{2}}\left(\xi^{(3,2)}\right)+b^{\xi_{3} \xi_{3}}\left(\xi^{(3,3)}\right)
\end{array}\right) .
\end{aligned}
$$


Furthermore, to compute $K_{F, c c}^{H}-K_{F, c f}^{H} T_{f c}^{H}$, we can take $(*)_{\beta, \alpha}$ with $\beta, \alpha \in$ $\{1,2,3\}$ by just replacing $\frac{\partial \widehat{\varphi}_{\alpha}^{L}}{\partial \xi_{\alpha}}$ by $\frac{\partial \hat{\varphi}_{\alpha}^{L}}{\partial \xi_{\alpha}}$. Evaluating the modified formulas, we obtain with (4.24)

$$
\widetilde{K}_{F, c c}^{L}=\frac{1}{2}\left(\begin{array}{ccc}
1 & 0 & 0 \\
0 & 1 & 0 \\
0 & 0 & 1
\end{array}\right)\left(\begin{array}{l}
b^{\xi_{1} \xi_{1}}\left(\xi^{(1,1)}\right)+b^{\xi_{2} \xi_{2}}\left(\xi^{(1,2)}\right) \\
b^{\xi_{1} \xi_{1}}\left(\xi^{(2,1)}\right)+b^{\xi_{3} \xi_{3}}\left(\xi^{(2,3)}\right) \\
b^{\xi_{2} \xi_{2}}\left(\xi^{(3,2)}\right)+b^{\xi_{3} \xi_{3}}\left(\xi^{(3,3)}\right)
\end{array}\right)
$$

which corresponds to the result when integration $K_{F, c c}^{L}$ by the nonstandard integration rule (4.8) on the macro element.

Since

$$
\widetilde{f}_{F}^{L}=T^{L^{T}} f_{F}^{H}=\left(\begin{array}{c}
f_{C}^{H}-T_{f c}^{H^{T}} f_{F}^{L} \\
f_{F}^{L}
\end{array}\right)
$$

we only have to consider the first part of the vector. Let us exemplarily consider $\alpha=1$. Then, we have, similar to (4.23), $\gamma \in\{4,6\}$ and

$$
\sum_{m=1}^{4} \int_{\Delta^{m}} \bar{f}\left(\varphi_{1}^{L_{C}}-\frac{1}{2} \sum_{\gamma \in\{4,6\}} \varphi_{\gamma}^{L}\right) \mathrm{d}\left(\xi_{1}, \xi_{2}\right) \approx \bar{f}\left(z_{1}\right) .
$$

which is equal to the approximation in $f_{F}^{L}$. As before we used $\bar{f}:=f|\operatorname{det} D F||\operatorname{det} D \widehat{F}| . \square$

We now provide a relation between the nodal and the hierarchical approach as provided in $[10,26]$ for the case of constant coefficients. For variable coefficient distributions, the provided integration formulas are not exact. The relation however also holds for varying coefficients. Note that transformations of the initial domain, such as polar coordinates, generally lead to nonconstant coefficients even if the coefficient in the initial equation, e.g., $\alpha(x, y)$ in (2.1), was constant.

THEOREM 4.3. For the stiffness matrices and right hand sides approximated by our (nonstandard) integration rules as described, we have

$$
\begin{aligned}
K_{F}^{L, e x} & =T^{L^{T}} K_{F}^{H, e x} T^{L}=T^{L^{T}} K_{F}^{P} T^{L}=K_{F}^{Q} \\
\text { and } \quad f_{F}^{L, e x} & =T^{L^{T}} f_{F}^{H, e x}=T^{L^{T}} f_{F}^{P}=f_{F}^{Q}
\end{aligned}
$$

Proof. In Lemma 4.2 we have shown that $K_{F}^{L}=\widetilde{K}_{F}^{L}$. With $T^{L}=\left(T^{H}\right)^{-1}$ it follows by using [11, Lemmata 3.1 and 3.2]

$$
K_{F}^{L, e x}=T^{L^{T}} K_{F}^{H, e x} T^{L}=T^{L^{T}} K_{F}^{P} T^{L} .
$$

The proof is complete by using Theorem 4.1.

REMARK 6 (Note on boundary conditions). Note that the implementation of Dirichlet boundary conditions and the symmetrization of the matrix can be done for the matrices on both levels (fine, $F$, and coarse, $C$ ) before combining the corresponding matrices and vectors to the extrapolated matrix and right hand side (4.10). This yields the same solution as in the case where the boundary conditions are implemented for the extra-polated matrix directly. However, both systems are not identical since the equations of boundary conditions on fine nodes are scaled by the factor $\frac{4}{3}$. 

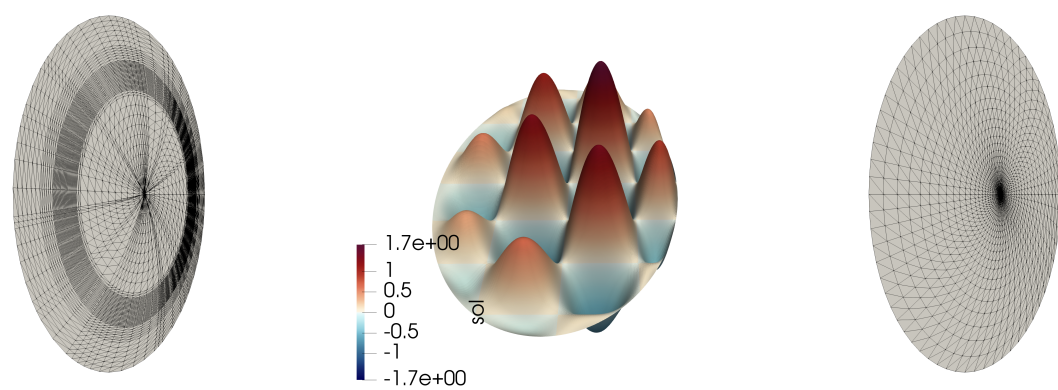

Figure 5.1. Geometry for for $\kappa=0.3, \delta=0.2$ and $r_{1}=10^{-6}$ with anisotropic grid for $\left(n_{r}, n_{\theta}\right)=(49,64)$ (left) and solution (5.3) (center). More regular grid with additional refinement around the origin for $\left(n_{r}, n_{\theta}\right)=(33,64)$ (right).

5. Numerical results. In this section, we consider the model problem (2.1) on $\Omega=F\left(\Omega_{\ell}\right)$ defined by

$$
\begin{array}{r}
(x, y)=F(r, \theta)=\left((1-\kappa) r \cos (\theta)-\delta r^{2},(1+\kappa) r \sin (\theta)\right) \\
\text { for }(r, \theta) \in \Omega_{\ell}=\left[r_{1}, 1.3\right] \times[0,2 \pi] ;
\end{array}
$$

where $r_{1}>0$; as introduced and then used in $[2,28]$ to describe tokamak cross-sections from fusion plasma applications. According to [2,28], we use $\kappa=0.3$ and $\delta=0.2$. We also provide results for $\kappa=\delta=0$ which results in a standard polar coordinate transformation. We use an anisotropic grid which is disturbed in $\theta$-direction and refined in $r$-direction such that $h_{\max } / h_{\min }=8$; cf. Figure 5.1 .

We use a typical density profile motivated from the application [27, 22], defined on the logical domain by

$$
\alpha(r, \theta)=\alpha(r)=\frac{2}{2.6+3.14}\left(1.3+\arctan \left(\frac{1-r}{0.09}\right)\right),
$$

i.e., here, the diffusion tensor $A: \mathbb{R}^{2} \rightarrow \mathbb{R}^{2}$ reduces to the function $\alpha: \mathbb{R} \rightarrow \mathbb{R}$.

Furthermore, we use the manufactured solution,

$$
u(x, y)=\left(1.3^{2}-r^{2}(x, y)\right) \cos (2 \pi x) \sin (2 \pi y) .
$$

We refrain from giving the right hand side $f$, which is a more than lengthy expression. We thank Edoardo Zoni for providing his Python script for the symbolic differentiation needed to compute the right hand side. The Dirichlet boundary conditions on $(r, \theta) \in$ $\left\{r_{1}, 1.3\right\} \times[0,2 \pi]$ are given accordingly.

Note that the two-level grids yield a natural set-up to use the presented extrapolation method in terms of a multigrid algorithm as presented in [10, 11]. In the current contribution, we will, however, build the matrix (4.10) for the extrapolated finite elements or differences explicitly. The interpretation as a multigrid method and the design of an efficient multigrid solver is left to the future work [14]. Here, all linear systems, in the standard or extrapolated approach, will be solved with a direct method.

5.1. Convergence results for tensor product structured grids. In this section, we consider two different geometries bounded away from the artificial singularity at $r_{1}=0.1$. On the (deformed) disks, we use a representative anisotropic, tensor product structured grid. 


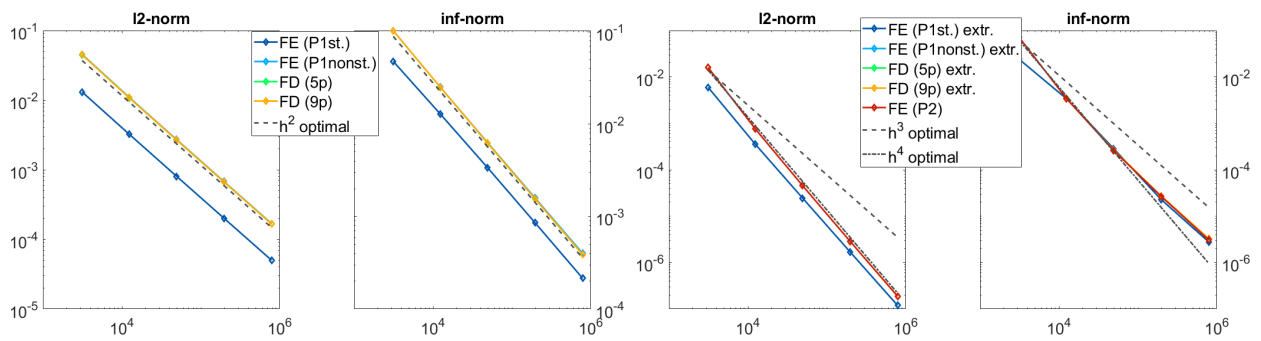

FIGURE 5.2. Error convergence in $\ell_{2}$ - and inf-norm for direct solution of finite difference and finite element discretizations (without extrapolation (left) and with extrapolation (right)) for $-\nabla \cdot(\alpha \nabla u)=f$ in $\Omega_{\ell}=(\mathbf{0 . 1}, 1.3) \times[0,2 \pi), f$ given by (5.3), $\Omega=F\left(\Omega_{\ell}\right)$ given by (5.1) with $\kappa=\delta=\mathbf{0}$ (i.e., standard polar coordinates), Dirichlet boundary conditions in $r$, periodic boundary conditions in $\theta$.

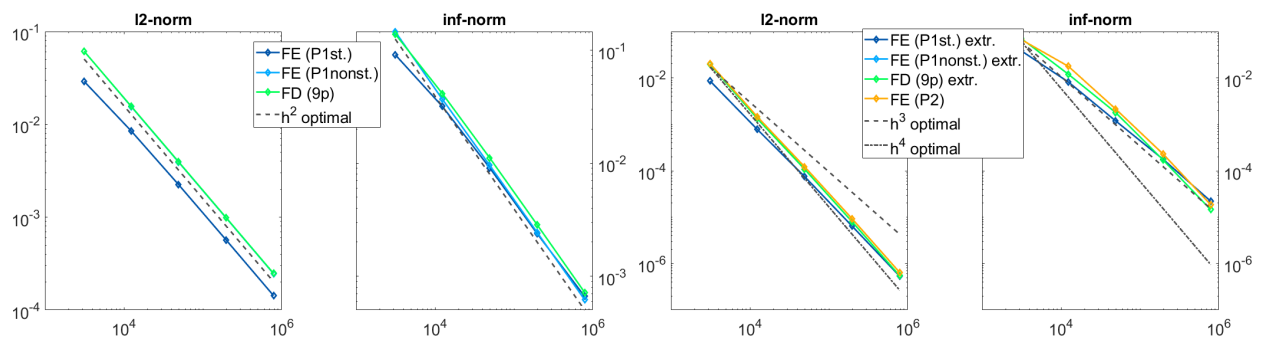

FIGURE 5.3. Error convergence in $\ell_{2}$ - and inf-norm for direct solution of finite difference and finite element discretizations (without extrapolation (left) and with extrapolation (right)) for $-\nabla \cdot(\alpha \nabla u)=f$ in $\Omega_{\ell}=(\mathbf{0 . 1}, 1.3) \times[0,2 \pi), f$ given by $(5.3), \Omega=F\left(\Omega_{\ell}\right)$ given by $(5.1)$ with $\kappa=\mathbf{0 . 3}$ and $\delta=\mathbf{0 . 2}$, Dirichlet boundary conditions in $r$, periodic boundary conditions in $\theta$.

We see that all our developed discretizations yield quadratic convergence for the circular as well as the deformed geometry; cf. Figure 5.2 and Figure 5.3.

If the extrapolation techniques as presented are used, i.e., if the stiffness matrices of two levels are combined, we obtain even fourth order convergence in $\ell_{2}$-norm for the circular geometry; cf. Figure 5.2. For the deformed geometry, the $\ell_{2}$-convergence is still much better than cubic but only almost of fourth order Figure 5.3. Depending on the geometry, the convergence in inf-norm is between third and fourth order. Despite the anisotropic mesh, the coefficient distribution, and the (deformed) geometry, we see that the convergence in $\ell_{2}$ norm is even (almost) quartic.

REMARK 7. The standard FE analysis predicts only third order for quadratic elements. However, we employ meshes that can be considered as created by a piecewise smooth transformation from a uniform mesh, so that certain symmetry conditions are approximately satisfied. Therefore we observe superconvergence effects. Note that extrapolation for symmetric finite differences on a uniform mesh would be expected to lead to fourth order accuracy, since only even orders appear in the asymptotic error expansion when the problem is regular enough. A detailed analysis of these effects will be an interesting direction for future research.

In order to directly highlight the differences in the geometries considered in Figure 5.2-5.5, we display the parameters that change in bold figure.

5.2. Convergence results for curvilinear coordinates when approaching the origin. In this section, we consider the additional difficulty introduced by ap- 


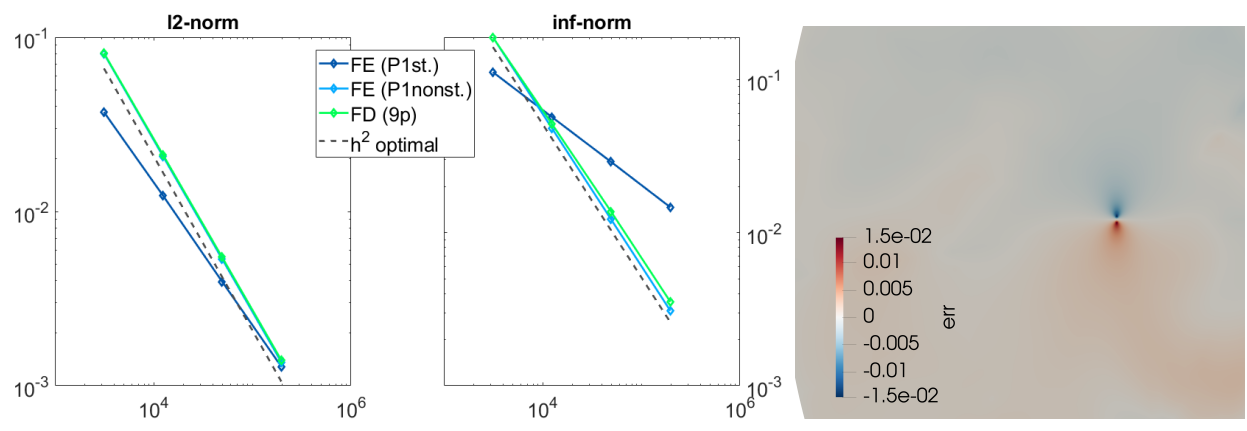

FIGURE 5.4. Left: Error convergence in $\ell_{2}$ - and inf-norm for direct solution of finite difference and finite element discretizations (without extrapolation) for $-\nabla \cdot(\alpha \nabla u)=f$ in $\Omega_{\ell}=\left(\mathbf{1 0}^{-\mathbf{6}}, 1.3\right) \times$

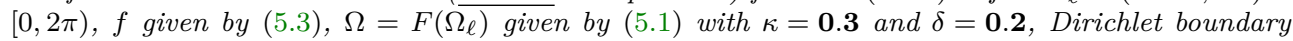
conditions in $r$, periodic boundary conditions in $\theta$. Right: Close-up of the error of FE (st), standard integration, for $\left(n_{r}, n_{\theta}\right)=(385,512)$ around the origin. Errors calculated by nodal comparison with exact solution.

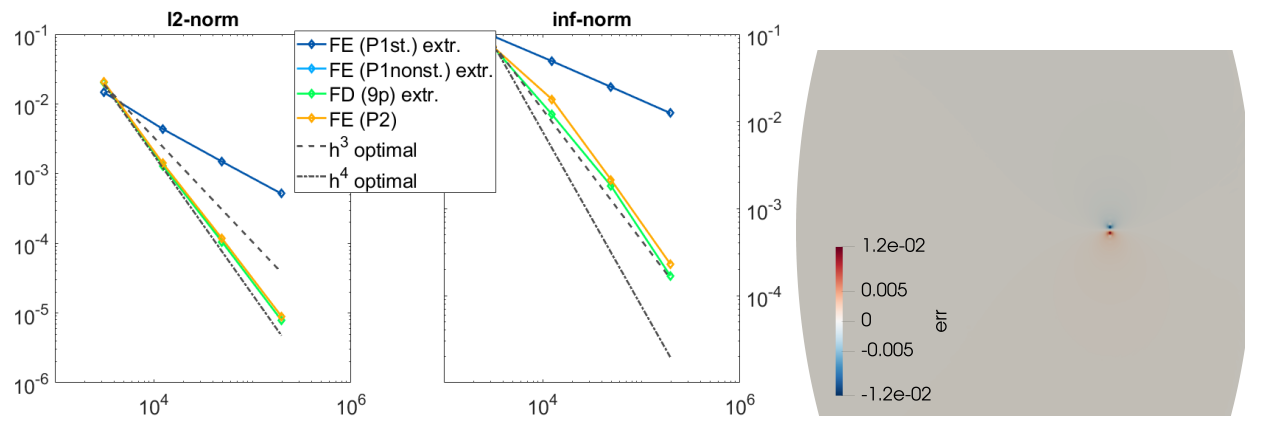

FIGURE 5.5. Left: Error convergence in $\ell_{2}$ - and inf-norm for direct solution of the two-level extrapolation method (4.10) combined with finite difference and finite element discretizations (with extrapolation for $F D$ and linear $F E)$ for $-\nabla \cdot(\alpha \nabla u)=f$ in $\Omega_{\ell}=\left(\mathbf{1 0}^{-\mathbf{6}}, 1.3\right) \times[0,2 \pi), f$ given by (5.3), $\Omega=F\left(\Omega_{\ell}\right)$ given by (5.1) with $\kappa=\mathbf{0 . 3}$ and $\delta=\mathbf{0 . 2}$, Dirichlet boundary conditions in $r$, periodic boundary conditions in $\theta$. Right: Close-up of the error of FE (st), standard integration, for $\left(n_{r}, n_{\theta}\right)=(385,512)$ around the origin. Errors calculated by nodal comparison with exact solution.

proaching the artificial singularity, i.e., we choose $r_{1}=10^{-6}$. We only consider the more complex, deformed geometry.

In Figure 5.4, we see that the standard integration for $\mathcal{P}_{1}$ elements does not lead to optimal convergence. Our nonstandard integration as well as the nine point stencil, on the other hand, yield qualitatively identitic results as in the case where $r_{1}=0.1$.

The suboptimal convergence of standard integration $\mathcal{P}_{1}$ elements in fact results from an insufficient approximation around the artificial singularity where the exact solution oscillates. It is possible to retrieve an optimal convergence behavior by refining the mesh towards the origin. In Table 5.1, we present the results for a mesh built according to [12], i.e. with nodes $r_{i}=r_{1}+\left(1.3-r_{1}\right)\left(i / n_{r}\right)^{3}, i=0, . ., n_{r}$, and uniform $\theta_{j}, j=1, . ., n_{\theta}$; see Figure 5.1 (right). 
TABLE 5.1

Error in $\ell_{2}$ - and inf-norm for direct solution of $P 1$ finite element discretization and its twolevel extrapolation method (4.10) discretizations for $-\nabla \cdot(\alpha \nabla u)=f$ in $\Omega_{\ell}=\left(\mathbf{1 0}^{-6}, 1.3\right) \times[0,2 \pi)$, $f$ given by (5.3), Dirichlet boundary conditions in $r$, periodic boundary conditions in $\theta . \Omega=F\left(\Omega_{\ell}\right)$ is discretized by an anisotropic mesh refined towards origin. Error reduction presented as ord.

\begin{tabular}{ll|cccccccc} 
& & \multicolumn{4}{|c}{ FE (P1st.) } & \multicolumn{4}{c}{ FE (P1st. extr.) } \\
$n_{r}$ & $n_{\theta}$ & $\|e r r\|_{l_{2}}$ & ord. & $\|e r r\|_{\infty}$ & ord. & $\|e r r\|_{l_{2}}$ & ord. & $\|$ err $\|_{\infty}$ & ord. \\
\hline 129 & 256 & $1.79 \mathrm{e}-03$ & - & $1.86 \mathrm{e}-02$ & - & $7.30 \mathrm{e}-05$ & - & $1.24 \mathrm{e}-03$ & - \\
257 & 512 & $4.67 \mathrm{e}-04$ & 1.94 & $4.96 \mathrm{e}-03$ & 1.91 & $5.29 \mathrm{e}-06$ & 3.80 & $1.14 \mathrm{e}-04$ & 3.45 \\
513 & 1024 & $1.18 \mathrm{e}-04$ & 1.99 & $1.26 \mathrm{e}-03$ & 1.98 & $3.63 \mathrm{e}-07$ & 3.88 & $9.42 \mathrm{e}-06$ & 3.61
\end{tabular}

6. Conclusion. We have presented finite difference discretizations maintaining, in a natural way, the symmetry of partial differential operators on anisotropic tensor product structured grids. In the absence of mixed terms $u_{r} u_{\theta}$ in the considered energy functional, quadratic convergence can be achieved with the presented five point stencil. Otherwise, nine point stencils should be used. We have also presented finite element approaches using nonstandard integration rules.

Furthermore, we have presented an extrapolation method combining grids with different discretization parameters to raise the convergence order. For the presented nonstandard finite element integration, we can provide a rigorous analysis. As predicted by the theory, the approach using nonstandard integration on linear elements resulted in the same discretization as a quadratic basis approach. In the experiments, the presented finite difference schemes behave similarly although lacking a rigorous proof. We obtain cubic convergence in the inf-norm for extrapolated finite element and finite difference discretizations and up to quartic convergence in $\ell_{2}$-norm.

A natural way to use our presented extrapolation technique are multigrid methods where the necessary two grid operators are inherently present. In fact, we believe this combination is one of the most efficient (in terms of memory requirements and floating point operations per second) techniques to reach higher order accuracy. For the combination of multigrid with our extrapolation technique, see [14].

Acknowledgement. The authors gratefully acknowledge the funding from the European Union's Horizon 2020 research and innovation programme under grant agreement no. 824158 .

\section{REFERENCES}

[1] M. I. Bakirova, V. A. Dorodnitsyn, and R. V. Kozlov, Symmetry-preserving difference schemes for some heat transfer equations, Journal of Physics A: Mathematical and General, 30 (1997), p. 8139, https://doi.org/10.1088/0305-4470/30/23/014.

[2] N. Bouzat, C. Bressan, V. Grandgirard, G. Latu, and M. Mehrenberger, Targeting Realistic Geometry in Tokamak Code Gysela, ESAIM: ProcS, 63 (2018), pp. 179-207, https://doi.org/10.1051/proc/201863179.

[3] A. Brandt and O. E. Livne, Multigrid techniques: 1984 guide with applications to fluid dynamics, vol. 67, SIAM, 2011, https://doi.org/10.1137/1.9781611970753.

[4] C. BREZINSKI AND M. R. ZaGLia, Extrapolation methods: theory and practice, vol. 2 of Studies in Computational Mathematics, Elsevier, Amsterdam Boston New York, 1991.

[5] R. DAI, P. Lin, AND J. Zhang, An efficient sixth-order solution for anisotropic Poisson equation with completed Richardson extrapolation and multiscale multigrid method, Computers \& Mathematics with Applications, 73 (2017), pp. 1865-1877, https://doi.org/10.1016/j. camwa.2017.02.020.

[6] V. Dorodnitsyn, Finite difference models entirely inheriting continuous symmetry of original differential equations, International Journal of Modern Physics C, 5 (1994), pp. 723-734, 
https://doi.org/10.1142/S012918319400083010.1007/978-94-011-2050-0_18.

[7] H. Egger, U. RüDE, And B. Wohlmuth, Energy-corrected finite element methods for corner singularities, SIAM Journal on Numerical Analysis, 52 (2014), pp. 171-193.

[8] V. Grandgirard, J. Abiteboul, J. Bigot, T. Cartier-Michaud, N. Crouseilles, G. DifPradalier, C. Ehrlacher, D. Esteve, X. Garbet, P. Ghendrih, G. Latu, M. MehrenBerger, C. Norscini, C. Passeron, F. Rozar, Y. SArazin, E. Sonnendrücker, A. Strugarek, And D. Zarzoso, A 5d gyrokinetic full-f global semi-lagrangian code for flux-driven ion turbulence simulations, Computer Physics Communications, 207 (2016), pp. 35 - 68, https://doi.org/https://doi.org/10.1016/j.cpc.2016.05.007.

[9] W. HACKBusch, Multigrid methods and applications, Springer, 1985, https://doi.org/10.1007/ 978-3-662-02427-0.

[10] M. JUNG AND U. RÜDE, Implicit extrapolation methods for multilevel finite element computations, SIAM Journal on Scientific Computing, 17 (1996), pp. 156-179, https: //doi.org/10.1137/0917012.

[11] M. JUNG AND U. RÜDE, Implicit extrapolation methods for variable coefficient problems, SIAM Journal on Scientific Computing, 19 (1998), pp. 1109-1124, https://doi.org/10.1137/ S1064827595293557.

[12] C. Kruse and M. Maischak, Convection problems on anisotropic meshes, Computational Methods in Applied Mathematics, 13 (2013), pp. 55-78, https://doi.org/10.1515/ cmam-2012-0003.

[13] M. J. Kühn, C. Kruse, And U. RÜDE, Energy-minimizing, symmetric finite differences for anisotropic meshes and energy functional extrapolation. Preprint, 2020, https://hal. archives-ouvertes.fr/hal-02941899.

[14] M. J. KÜhn, C. Kruse, And U. RÜDE, Extrapolated geometric multigrid on disk-like domains for the gyrokinetic Poisson equation from fusion plasma applications, (2020), https://hal. archives-ouvertes.fr/hal-03003307. Preprint.

[15] J. Lyness And U. RÜDE, Cubature of integrands containing derivatives, Numerische Mathematik, 78 (1998), pp. 439-461, https://doi.org/10.1007/s002110050320.

[16] G. Marchuk and V. Shaidurov, Difference methods and their extrapolations, Springer-Verlag, New York, 1983, https://doi.org/10.1007/978-1-4613-8224-9.

[17] K. PAN, D. He, AND H. Hu, An extrapolation cascadic multigrid method combined with a fourth-order compact scheme for 3D Poisson equation, Journal of Scientific Computing, 70 (2017), pp. 1180-1203, https://doi.org/10.1007/s10915-016-0275-9.

[18] U. RÜDE, Local corrections for eliminating the pollution effect of reentrant corners, Tech. Report TUM-INFO-02-89-I-01-350-1-FMI, Bibliothek der Fakultät für Mathematik und Informatik, TU München, 1989.

[19] U. RÜDE, Extrapolation and related techniques for solving elliptic equations, Tech. Report TUM-I9135, Institut für Informatik, TU München, 1991. http://citeseerx.ist.psu.edu/ viewdoc/summary?doi=10.1.1.16.9471.

[20] U. RÜDE, The hierarchical basis extrapolation method, SIAM Journal on Scientific and Statistical Computing, 13 (1992), pp. 307-318, https://doi.org/10.1137/0913016.

[21] Y. SAAD, Iterative Methods for Sparse Linear Systems, Society for Industrial and Applied Mathematics, Philadelphia, PA, USA, 2nd ed., 2003, https://doi.org/10.1137/1.9780898718003.

[22] E. Sonnendrücker, 2019. Private communication.

[23] S. Tikhovskaya, Solving a singularly perturbed elliptic problem by a cascadic multigrid algorithm with Richardson extrapolation, in International Conference on Finite Difference Methods, Springer, 2018, pp. 533-541, https://doi.org/10.1007/978-3-030-11539-5_62.

[24] B. van Es, B. Koren, and H. J. De Blank, Finite-difference schemes for anisotropic diffusion, Journal of Computational Physics, 272 (2014), pp. 526 - 549, https://doi.org/10.1016/j. jcp.2014.04.046.

[25] R. S. VArga, Matrix Iterative Analysis, Prentice-Hall, Englewood Cliffs, NJ., 1962, https: //doi.org/10.1007/978-3-642-05156-2.

[26] H. Yserentant, On the multi-level splitting of finite element spaces, Numerische Mathematik, 49 (1986), pp. 379-412, https://doi.org/10.1007/BF01389538.

[27] E. Zoni, Theoretical and numerical studies of gyrokinetic models for shaped tokamak plasmas, PhD thesis, Technische Universität München, München, 2019.

[28] E. ZONI AND Y. GüÇLÜ, Solving hyperbolic-elliptic problems on singular mapped disk-like domains with the method of characteristics and spline finite elements, Journal of Computational Physics, 398 (2019), p. 108889, https://doi.org/10.1016/j.jcp.2019.108889. 
7. Appendix. For completeness, we provide all the necessary basis functions on the different reference element representations,

$$
\begin{aligned}
& \widehat{\varphi}_{1}^{L}= \begin{cases}1-2 \xi_{1}-2 \xi_{2}, & \text { in } \Delta^{1}, \\
0, & \text { otherwise, }\end{cases} \\
& \widehat{\varphi}_{2}^{L}= \begin{cases}-1+2 \xi_{1}, & \text { in } \Delta^{2}, \\
0, & \text { otherwise, }\end{cases} \\
& \widehat{\varphi}_{3}^{L}= \begin{cases}-1+2 \xi_{2}, & \text { in } \Delta^{3}, \\
0, & \text { otherwise, }\end{cases} \\
& \widehat{\varphi}_{4}^{H}=\widehat{\varphi}_{4}^{L}= \begin{cases}2 \xi_{1}, & \text { in } \Delta^{1}, \\
2-2 \xi_{1}-2 \xi_{2}, & \text { in } \Delta^{2}, \\
0, & \text { in } \Delta^{3}, \\
1-2 \xi_{2}, & \text { in } \Delta^{4},\end{cases} \\
& \widehat{\varphi}_{5}^{H}=\widehat{\varphi}_{5}^{L}= \begin{cases}0, & \text { in } \Delta^{1}, \\
2 \xi_{2}, & \text { in } \Delta^{2}, \\
2 \xi_{1}, & \text { in } \Delta^{3}, \\
2 \xi_{1}+2 \xi_{2}-1, & \text { in } \Delta^{4},\end{cases} \\
& \widehat{\varphi}_{6}^{H}=\widehat{\varphi}_{6}^{L}= \begin{cases}2 \xi_{2}, & \text { in } \Delta^{1}, \\
0, & \text { in } \Delta^{2}, \\
2-2 \xi_{1}-2 \xi_{2}, & \text { in } \Delta^{3}, \\
1-2 \xi_{1}, & \text { in } \Delta^{4},\end{cases} \\
& \widehat{\varphi}_{1}^{Q}=2 \xi_{1}^{2}+2 \xi_{2}^{2}-3 \xi_{1}-3 \xi_{2}+4 \xi_{1} \xi_{2}+1, \text { in } T, \\
& \widehat{\varphi}_{2}^{Q}=2 \xi_{1}^{2}-\xi_{1}, \text { in } T \text {, } \\
& \widehat{\varphi}_{3}^{Q}=2 \xi_{2}^{2}-\xi_{2}, \text { in } T \text {, } \\
& \widehat{\varphi}_{4}^{P}=\widehat{\varphi}_{4}^{Q}=4 \xi_{1}\left(1-\xi_{1}-\xi_{2}\right) \text {, in } T \text {, } \\
& \widehat{\varphi}_{5}^{P}=\widehat{\varphi}_{5}^{Q}=4 \xi_{1} \xi_{2} \text {, in } T \text {, } \\
& \widehat{\varphi}_{6}^{P}=\widehat{\varphi}_{6}^{Q}=4 \xi_{2}\left(1-\xi_{1}-\xi_{2}\right) \text {, in } T \text {, } \\
& \widehat{\varphi}_{1}^{H}=\widehat{\varphi}_{1}^{P}=\widehat{\varphi}_{1}^{L_{C}}=1-\xi_{1}-\xi_{2}, \text { in } T, \\
& \widehat{\varphi}_{2}^{H}=\widehat{\varphi}_{2}^{P}=\widehat{\varphi}_{2}^{L_{C}}=\xi_{1} \text {, in } T \text {, } \\
& \widehat{\varphi}_{3}^{H}=\widehat{\varphi}_{3}^{P}=\widehat{\varphi}_{3}^{L_{C}}=\xi_{2} \text {, in } T \text {, }
\end{aligned}
$$

as well as the nodal evaluations of the directional derivatives used for the nonstandard numerical integration in the following Table 7.1. 


\begin{tabular}{|c|c|c|c|c|c|c|c|c|c|c|c|c|c|c|c|c|c|}
\hline & $\mathrm{T}$ & $\Delta^{1}$ & 2 & $\Delta^{3}$ & $\Delta^{4}$ & & $\mathrm{~T}$ & $\Delta^{1}$ & $\Delta^{2}$ & $\Delta^{3}$ & $\Delta^{4}$ & & $\mathrm{~T}$ & $\Delta^{1}$ & $\Delta^{2}$ & $\Delta^{3}$ & $\Delta^{4}$ \\
\hline & \multicolumn{5}{|c|}{$\xi^{(T, 1)} \xi^{(1,1)} \xi^{(2,1)} \xi^{(3,1)} \xi^{(4,1)}$} & & \multicolumn{5}{|c|}{$\xi^{(T, 2)} \xi^{(1,2)} \xi^{(2,2)} \xi^{(3,2)} \xi^{(4,2)}$} & & \multicolumn{5}{|c|}{$\xi^{(T, 3)} \xi^{(1,3)} \xi^{(2,3)} \xi^{(3,3)} \xi^{(4,3)}$} \\
\hline$\frac{\partial \widehat{\varphi}_{1}^{L}}{\partial \xi_{1}}$ & - & -2 & 0 & 0 & 0 & $\frac{\partial \widehat{\varphi}_{1}^{L}}{\partial \xi_{2}}$ & - & -2 & 0 & 0 & 0 & $\frac{\partial \widehat{\varphi}_{1}^{L}}{\partial \xi_{3}}$ & - & 0 & 0 & 0 & 0 \\
\hline$\frac{\partial \widehat{\varphi}_{1}^{Q}}{\partial \xi_{1}}$ & -1 & -2 & 0 & 0 & 0 & $\frac{\partial \widehat{\varphi}_{1}^{Q}}{\partial \xi_{2}}$ & -1 & -2 & 0 & 0 & 0 & $\frac{\partial \widehat{\varphi}_{1}^{Q}}{\partial \xi_{3}}$ & 0 & 0 & 0 & 0 & 0 \\
\hline$\frac{\partial \widehat{\varphi}_{1}^{L_{C}}}{\partial \xi_{1}}$ & -1 & -1 & -1 & -1 & -1 & $\frac{\partial \widehat{\varphi}_{1}^{L_{C}}}{\partial \xi_{2}}$ & -1 & -1 & -1 & -1 & -1 & $\frac{\partial \widehat{\varphi}_{1}^{L_{C}}}{\partial \xi_{3}}$ & 0 & 0 & 0 & 0 & 0 \\
\hline$\frac{\partial \widehat{\varphi}_{2}^{L}}{\partial \xi_{1}}$ & - & 0 & 2 & 0 & 0 & $\frac{\partial \widehat{\varphi}_{2}^{L}}{\partial \xi_{2}}$ & - & 0 & 0 & 0 & 0 & $\frac{\partial \widehat{\varphi}_{2}^{L}}{\partial \xi_{3}}$ & - & 0 & -2 & 0 & 0 \\
\hline$\frac{\partial \widehat{\varphi}_{2}^{Q}}{\partial \xi_{1}}$ & 1 & 0 & 2 & 0 & 0 & $\frac{\partial \widehat{\varphi}_{2}^{Q}}{\partial \xi_{2}}$ & 0 & 0 & 0 & 0 & 0 & $\frac{\partial \widehat{\varphi}_{2}^{Q}}{\partial \xi_{3}}$ & -1 & 0 & -2 & 0 & 0 \\
\hline$\frac{\partial \widehat{\varphi}_{2}^{L_{C}}}{\partial \xi_{1}}$ & 1 & 1 & 1 & 1 & 1 & $\frac{\partial \widehat{\varphi}_{2}^{L_{C}}}{\partial \xi_{2}}$ & 0 & 0 & 0 & 0 & 0 & $\frac{\partial \widehat{\varphi}_{2}^{L} C}{\partial \xi_{3}}$ & -1 & -1 & -1 & -1 & -1 \\
\hline$\frac{\partial \widehat{\varphi}_{3}^{L}}{\partial \xi_{1}}$ & - & 0 & 0 & 0 & 0 & $\frac{\partial \widehat{\varphi}_{3}^{L}}{\partial \xi_{2}}$ & - & 0 & 0 & 2 & 0 & $\frac{\partial \widehat{\varphi}_{3}^{L}}{\partial \xi_{3}}$ & - & 0 & 0 & 2 & 0 \\
\hline$\frac{\partial \widehat{\varphi}_{3}^{Q}}{\partial \xi_{1}}$ & 0 & 0 & 0 & 0 & 0 & $\frac{\partial \widehat{\varphi}_{3}^{Q}}{\partial \xi_{2}}$ & 1 & 0 & 0 & 2 & 0 & $\frac{\partial \widehat{\varphi}_{3}^{Q}}{\partial \xi_{3}}$ & 1 & 0 & 0 & 2 & 0 \\
\hline$\frac{\partial \widehat{\varphi}_{3}^{L_{C}}}{\partial \xi_{1}}$ & 0 & 0 & 0 & 0 & 0 & $\frac{\partial \widehat{\varphi}_{3}^{L_{C}}}{\partial \xi_{2}}$ & 1 & 1 & 1 & 1 & 1 & $\frac{\partial \widehat{\varphi}_{3}^{L_{C}}}{\partial \xi_{3}}$ & 1 & 1 & 1 & 1 & 1 \\
\hline$\frac{\partial \widehat{\varphi}_{4}^{L}}{\partial \xi_{1}}$ & - & 2 & -2 & 0 & 0 & $\frac{\partial \widehat{\varphi}_{4}^{L}}{\partial \xi_{2}}$ & - & 0 & -2 & 0 & -2 & $\frac{\partial \widehat{\varphi}_{4}^{L}}{\partial \xi_{3}}$ & - & -2 & 0 & 0 & -2 \\
\hline$\frac{\partial \widehat{\varphi}_{5}^{L}}{\partial \xi_{1}}$ & - & 0 & 0 & 2 & 2 & $\frac{\partial \widehat{\varphi}_{5}^{L}}{\partial \xi_{2}}$ & - & 0 & 2 & 0 & 2 & $\frac{\partial \widehat{\varphi}_{5}^{L}}{\partial \xi_{3}}$ & - & 0 & 2 & -2 & 0 \\
\hline$\frac{\partial \widehat{\varphi}_{6}^{L}}{\partial \xi_{1}}$ & - & 0 & 0 & -2 & -2 & $\frac{\partial \widehat{\varphi}_{6}^{L}}{\partial \xi_{2}}$ & - & 2 & 0 & -2 & 0 & $\frac{\partial \widehat{\varphi}_{6}^{L}}{\partial \xi_{3}}$ & - & 2 & 0 & 0 & 2 \\
\hline$\frac{\partial \widehat{\varphi}_{4}^{Q}}{\partial \xi_{1}}$ & 0 & 2 & -2 & 0 & 0 & $\frac{\partial \widehat{\varphi}_{4}^{Q}}{\partial \xi_{2}}$ & 0 & 0 & -2 & 0 & -2 & $\frac{\partial \widehat{\varphi}_{4}^{Q}}{\partial \xi_{3}}$ & 0 & -2 & 0 & 0 & -2 \\
\hline$\frac{\partial \widehat{\varphi}_{5}^{Q}}{\partial \xi_{1}}$ & 0 & 0 & 0 & 2 & 2 & $\frac{\partial \widehat{\varphi}_{5}^{Q}}{\partial \xi_{2}}$ & 0 & 0 & 2 & 0 & 2 & $\frac{\partial \widehat{\varphi}_{5}^{Q}}{\partial \xi_{3}}$ & 0 & 0 & 2 & -2 & 0 \\
\hline$\frac{\partial \widehat{\varphi}_{6}^{Q}}{\partial \xi_{1}}$ & 0 & 0 & 0 & -2 & -2 & $\frac{\partial \widehat{\varphi}_{6}^{Q}}{\partial \xi_{2}}$ & 0 & 2 & 0 & -2 & 0 & $\frac{\partial \widehat{\varphi}_{6}^{Q}}{\partial \xi_{3}}$ & 0 & 2 & 0 & 0 & 2 \\
\hline
\end{tabular}

TABLE 7.1

Values of the directional derivatives of $\widehat{\varphi}_{k}^{L_{C}}, k \in\{1,2,3\}$, and $\widehat{\varphi}_{k}^{L}, \widehat{\varphi}_{k}^{Q}, k \in\{1, \ldots, 6\}$, at evaluation nodes $\xi^{(T, n)}$ and $\xi^{(m, n)}, m \in\{1,2,3,4\}, n \in\{1,2,3\}$; cf. Figure 4.1 and Figure 4.2. Nonexisting directional derivatives are marked by a dash (-). These entries are however not needed in the nonstandard numerical integration. 